\title{
ZOOARQUEOLOGÍA DE LOS MACROVERTEBRADOS DEL YACIMIENTO FENICIO DEL TEATRO CÓMICO (CÁDIZ)
}

\author{
MACROVERTEBRATE ZOOARCHAEOLOGY OF THE \\ PHOENICIAN SITE OF TEATRO CÓMICO (CÁDIZ)
}

\author{
VERÓNICA ESTACA GÓMEZ* \\ JOSÉ YRAVEDRA SAINZ DE LOS TERREROS* \\ JOSÉ $\mathrm{M}^{\mathrm{a}}$ GENER BASALLOTE** \\ MARÍA DE LOS ÁNGELES NAVARRO GARCÍA** \\ JUAN MIGUEL PAJUELO SÁEZ** \\ MARIANO TORRES ORTIZ*
}

\begin{abstract}
Resumen: En este trabajo presentamos el estudio zooarqueológico de los macromamíferos del yacimiento de época fenicia del Teatro Cómico. Los ovicápridos son la cabaña ganadera más importante seguidos del vacuno y el porcino según el periodo de ocupación. A continuación están representados el caballo y el perro, aunque con porcentajes pequeños. En cuanto a la fauna silvestre su representación es baja, destacando entre ellos el ciervo. Los datos taxonómicos, esqueléticos, y los patrones de mortandad, unidos al registro arqueológico, nos permiten concluir que las especies domésticas tuvieron una funcionalidad orientada a distintos usos económicos como la producción de leche y lana, lo cual es acorde a lo observado en otros yacimientos de este momento
\end{abstract} Palabras clave: Zooarqueología, patrones de mortandad, usos económicos, época fenicia, Gadir

\footnotetext{
* Departamento de Prehistoria de la Universidad Complutense de Madrid. Prof. Aranguren, 28040. Madrid. Correo-e: vestacag@ hotmail.com

** Departamento de Prehistoria de la Universidad Complutense de Madrid. Prof. Aranguren, 28040. Madrid. Correo-e: joyravedra@ hotmail.com
}

\begin{abstract}
This paper presents the zooarchaeological study of the macromammals recovered at the Phoenician site called Teatro Cómico. Ovicaprids constitute the main herding followed by cattle and porcine, depending on the occupation period. Other species, with a reduced representation, are horse and dog. Regarding wild fauna, a low presence was recorded, deer being the most significant species. Taxonomic and skeletal and death-patterns data, supplemented by the archaeological record, lead to the conclusion that the rearing of domestic species was oriented towards diverse economic uses such as milk and wool production, in line with contemporaneous records in other sites.
\end{abstract}

Key-words: Zooarchaeology, death patterns, economic use, Phoenician period, Gadir

*** Área de Urbanismo del Excmo. Ayuntamiento de Cádiz. Plaza de San Juan de Dios sn, 11005, Cádiz. Correo-e: generarqu@ hotmail.com

**** Departamento de Prehistoria de la Universidad Complutense de Madrid. Prof. Aranguren, 28040. Madrid. Correo-e: mtorreso@ghis. ucm.es 


\section{INTRODUCCIÓN}

Hasta el momento, los estudios zooarqueológicos en el ámbito del mundo fenicio han sido en su conjunto bastante escasos en proporción al número de yacimientos excavados, a pesar de que desde momentos muy tempranos se hicieran algunos trabajos (Martín Roldán 1959). La causa de esto está relacionada con una concepción clásica de la arqueología, que solo analiza o recoge las evidencias materiales más significativas de la cultura humana, entre las que destacan la cerámica y la industria lítica. La consecuencia de esta forma de hacer arqueología es la ausencia de proyectos interdisciplinares y el desprecio por la arqueología que estudia el contexto. Aunque este problema no es único en el mundo fenicio (Yravedra y Estaca 2013), en nuestro caso sólo nos referiremos a los estudios de los yacimientos fenicios del sur de la península ibérica. La escasez de análisis faunísticos pertenecientes a época fenicia ha sido ya planteada por Riquelme (2001) y otros investigadores (Uerpmann y Uerpmann 1973; Morales et al. 1994, 1995; Montero 1999). En este trabajo se presentarán los datos zooarqueológicos del Teatro Cómico que se sumarán a los datos conocidos de otras estaciones fenicias y tartésicas como Monte Berrueco, Campillo, Pocito Chico (Estévez 1985; Riquelme 2001b) y el Castillo de Doña Blanca (Morales et al. 1994, 1995) en Cádiz, el Cerro Villar (Montero 1999), San Agustín (Bernáldez 1990) y Toscanos (Uerpmann y Uerpmann 1973) en Málaga y Montemolín (De la Bandera et al. 1995; Chaves et al. 2000), Carmona (Bernáldez 1997), Calle Alcazaba de Lebrija (Bernáldez 1999; Bernáldez y Bernáldez 2000), San Isidro 85-6 (Bernáldez 1988), el Carambolo (Marín Roldán 1959) y Setefilla (Estévez 1983) en Sevilla.

\section{EL TEATRO CÓMICO. EL CONTEXTO ESTRATIGRÁFICO DE LOS RESTOS ÓSEOS ESTUDIADOS}

El yacimiento arqueológico del Teatro Cómico se encuentra situado en el actual casco histórico de la ciudad de Cádiz. Su ubicación topográfica corresponde a la zona más alta de la antigua Erytheia, la isla menor del antiguo archipiélago de las Gadeiras y donde muchos investigadores han situado el primitivo asentamiento urbano de Gadir (Fierro 1979; 1983; 1995: 101-116; Ramírez 1982: 63, 85 104, 140; Escacena 1985: 43) (fig. 1).

En las intervenciones arqueológicas promovidas desde el año 2002 por el Ayuntamiento de Cádiz se han delimitado diez momentos de ocupación (Gener et al. 2012; Zamora et al. 2010) (fig. 2). A continuación, mostraremos el análisis zooarqueológico de los macrovertebrados hallados en los distintos periodos del asentamiento urbano de época fenicia del Cómico, con el fin de obtener nuevas informaciones sobre los modos de vida de las ciudades protohistóricas.

\subsection{Periodo II: Fenicio A (circa $820 / 800-720$ a.C.)}

Las construcciones de este periodo se organizan en tres grupos estructurales, articulados por dos calles en las que se diferencian ocho unidades domésticas (viviendas). Fueron construidos siguiendo los parámetros de la arquitectura de tierra. Todo este conjunto experimentó diversas reestructuraciones a lo largo de cuatro fases, incluyendo modificaciones en las calles.

Estamos ante una trama compacta de volúmenes cuboides escalonados, colindantes y adyacentes, originados posiblemente por una alta densidad poblacional. Se distribuía en terrazas que descendían hacia la orilla del canal Bahía-Caleta y se articulaba a partir de calles que iban sorteando los cambios de rasante. Esta ordenación agrupada debía de seguir un modelo basado fundamentalmente en la proximidad y en la participación social de unos parámetros constructivos y organizativos comunes, pero sin la rigidez imperativa de los modelos clásicos. Las calles eran espacios comunes de tránsito, probablemente consideradas ejes articuladores y, hasta cierto punto, respetadas urbanísticamente, pues no solo se preservaron a pesar de las reformas de los edificios sino que además se mantuvieron y se repavimentaron. Todo ello indica una actividad comunitaria propia de una ciudad, donde el espacio público tiene cierta prioridad sobre el privado, tal y como ocurre en muchos asentamientos orientales (Herzog 2007, para Israel; Hellas y Marzoli 2009, para el urbanismo fenicio en general; Yasur-Landau, Ebeling y Mazow 2011, para urbanismo oriental en general; Fumadó 2013, para el caso de Cartago; Gener et al. e.p.) (figs. 3, 4, 5).

En este ámbito urbano han quedado grabadas algunas improntas del tránsito de animales y personas en las numerosas huellas conservadas en el pavimento de una de las calles, en el que se observa el paso de bóvidos y ovicápridos (fig. 5).

Los edificios estaban organizados en varios complejos o estancias con distintos usos domésticos y artesanales, como por ejemplo talleres de alfarería, almacenes, etc. 


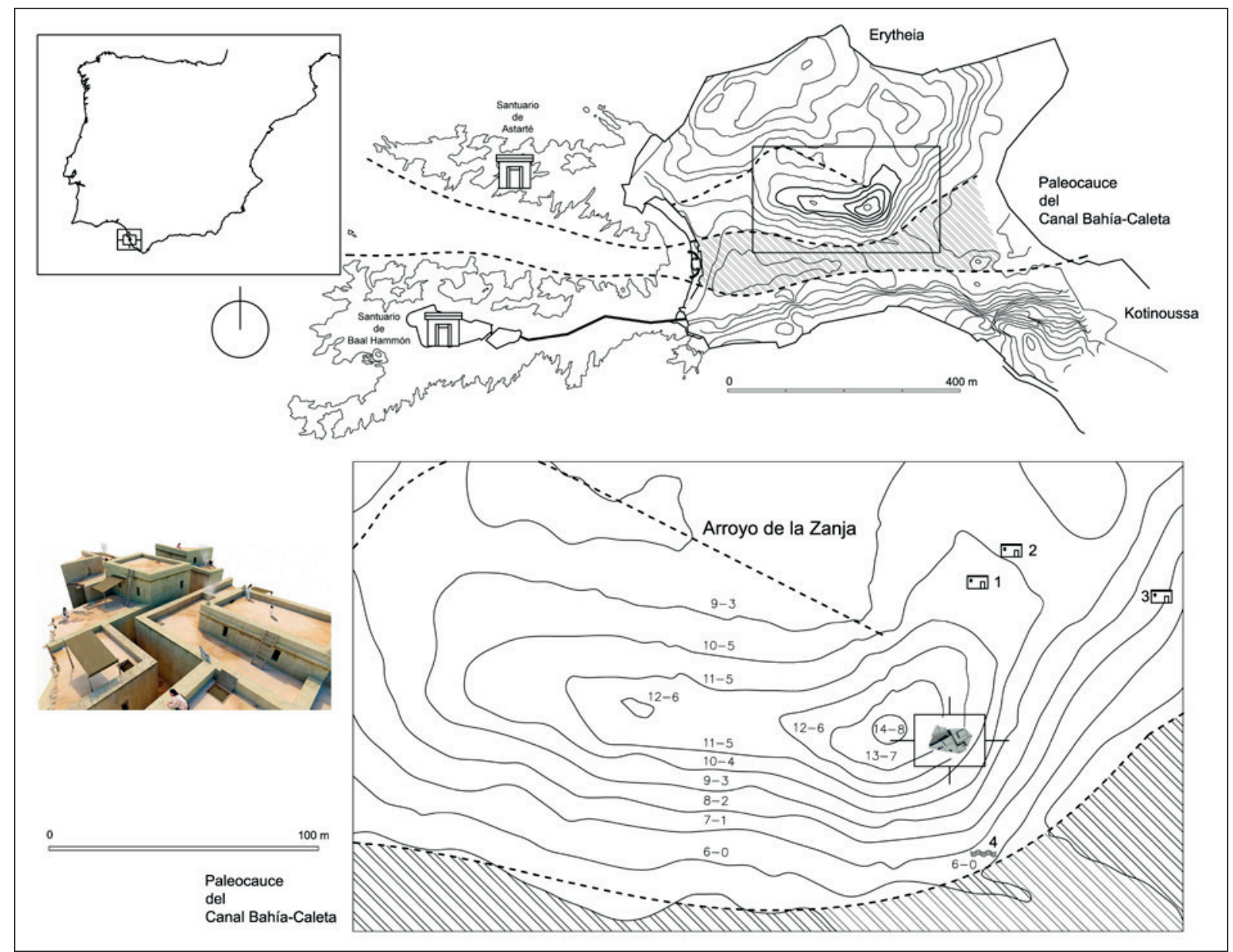

Figura 1. Ubicación topográfica del Teatro Cómico: 1.- C/ Ancha n 29; 2.- C/ Central de Teléfonos C/Ancha; 3.- C/ Cánovas del Castillo n ${ }^{\circ} 38$; 4.- Paleo-playa documentada en el solar del antiguo Teatro de Andalucía (J.Mª.G.).

\subsection{Periodo III: Fenicio B (mediados del siglo VIII hasta inicios del siglo VI a.C.)}

A diferencia de las viviendas anteriores, los edificios de este momento fueron realizados con mampostería de piedra ostionera y arcilla roja. La piedra ostionera es el nombre común de un conglomerado o lumaquela bioclástica, de base silícea, originada por los depósitos del Plioceno Superior-Pliocuaternario, compuesta de lamelibranquios (Ostreas y pectínidos) y cantos rodados de cuarcita y cuarzo principalmente (Gutiérrez et al. 1991: 101-109; Domínguez-Bella 2011: 62-68). La denominación local "arcilla roja" se refiere al producto creado de la decantación y preparación de las "arenas rojas" aluviales, un depósito fluvio-marino continental formado en el tránsito Neógeno-Cuaternario. Se trata de una formación arenosa de aspecto masivo con cantos de cuarcita, cuarzo, filitas, etc., depositada directamente sobre el sustrato rocoso (lumaquela bioclástica) que suele aparecer karstificado (Domínguez-Bella 2011: 62).

La estructura mejor conservada está levantada con aparejo de pilares. Este tipo de aparejo se considera una evolución de una técnica constructiva bien conocida en el Próximo Oriente, la pier-and-rubble-masonry, que en Occidente evoluciona sustituyéndose los pilares construidos de sillares bien escuadrados por un único bloque u ortostato de piedra dispuesto verticalmente en el paramento (Elayi 1990, 1996; Sharon 1987). El único grupo estructural excavado de esta fase tiene al menos cinco estancias.

Al igual que en el periodo anterior, la desaparición de este edificio fue provocada por un acontecimiento traumático según indican dos cadáveres documentados in situ (Calero et al. 2012; Calero et al. e.p.). 


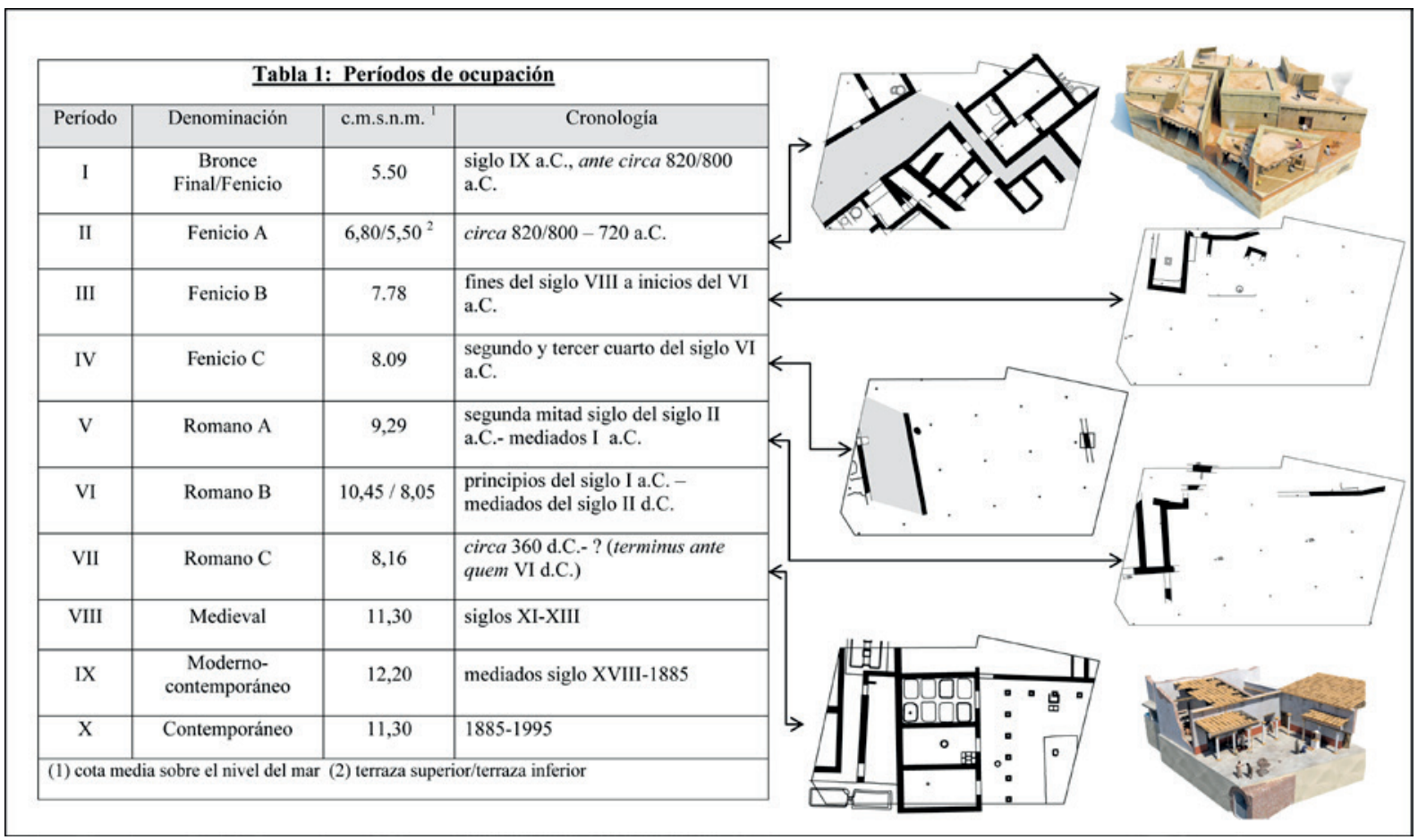

Figura 2. Periodos de ocupación documentados en el solar del antiguo Teatro Cómico.

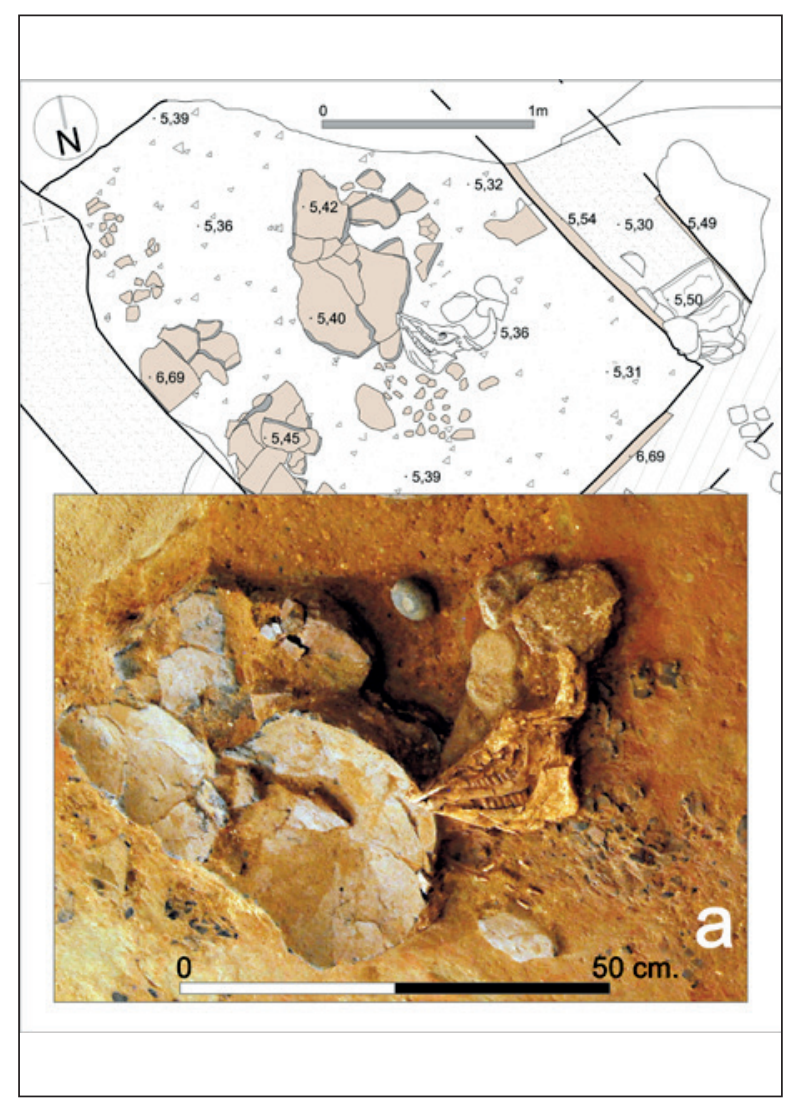

\subsection{Periodo IV: Fenicio C (segundo y tercer cuarto del siglo VI a.C.)}

Toda la zona se reurbanizó desmantelándose parcialmente las construcciones abandonadas, volviéndose a nivelar el terreno para levantar dos nuevos edificios separados por una calle de casi $5 \mathrm{~m}$ de anchura. Este espacio de tránsito fue pavimentado con una arcilla menos compacta que la de los periodos anteriores. Desgraciadamente, las estructuras están muy afectadas por las construcciones de época romana, por lo que solo se conservan parte de los muros de fachada, algunos restos de pavimento de arcilla apisonada, un umbral con pavimento de conchas de diferentes especies y la base de un hogar fabricado con una torta de arcilla cubierta con fragmentos de cerámica común. A partir de los datos obtenidos, se puede considerar que el hecho más sustancial es el cambio del trazado urbanístico, pues el antiguo eje suroeste-nordeste es sustituido por otro con una orientación exacta norte-sur.

Figura 3. Cráneo de bóvido encontrado en una de las habitaciones utilizada como almacén en la unidad doméstica n 4 (J.M. G y J.M.P.). 


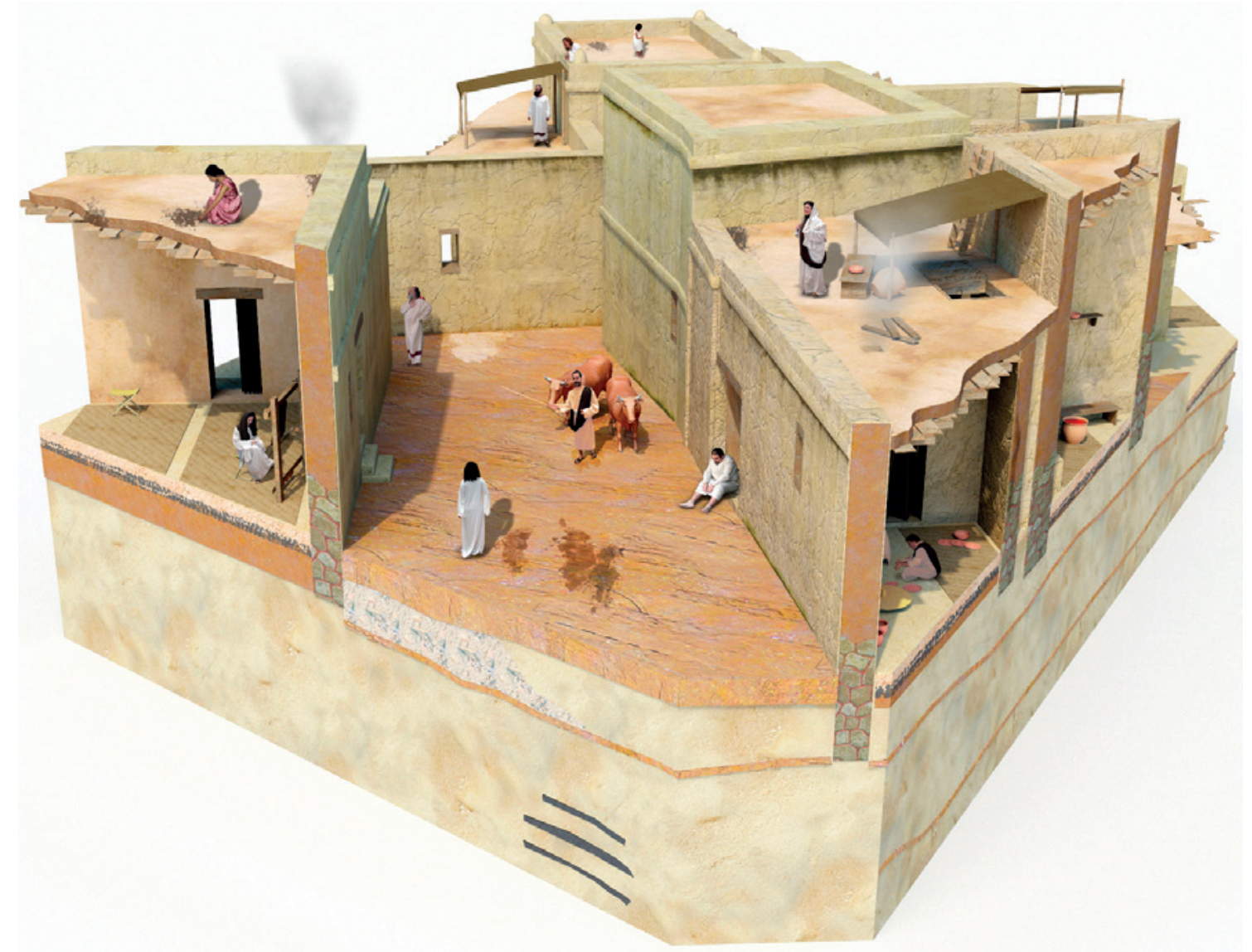

Figura 4. Propuesta de reconstrucción en 3D de las viviendas del periodo II (Fenicio A) (J.M.G. y Gesdata, S.L.).

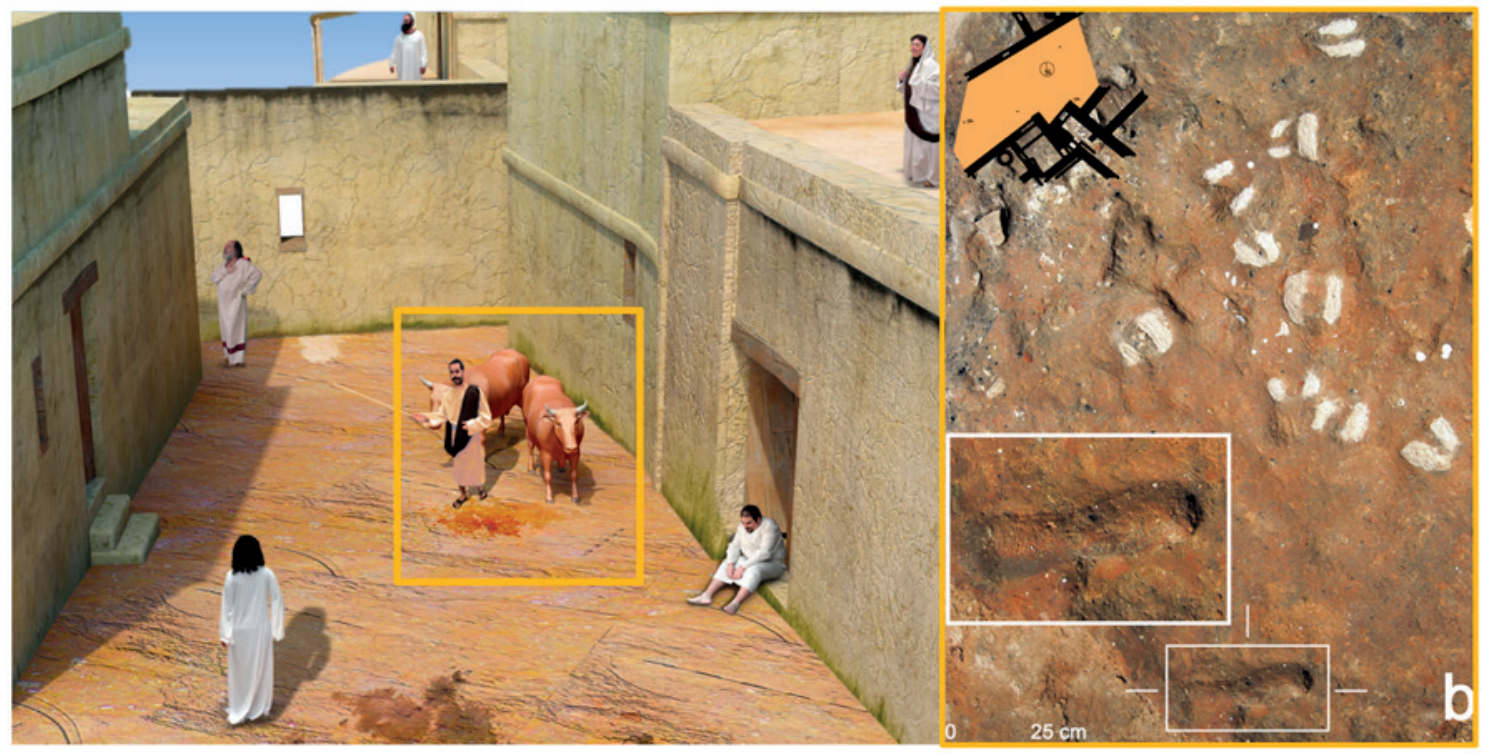

Figura 5. Izq.: Detalle de la reconstrucción en 3D de una de las calles. Der.: Huellas de bóvidos documentadas en el pavimento de la calle $\mathrm{n}^{\mathrm{o}} 1$. En el inferior se observa una posible huella humana (J.M.G. y Gesdata, S.L.). 


\section{MATERIALES Y MÉTODOS}

\subsection{Materiales}

En este trabajo presentamos los datos relativos a los macromamíferos del Teatro Cómico. La muestra ósea analizada asciende a 1.719 restos, mostrando unas buenas condiciones de conservación y una elevada fragmentación. Las especies identificadas son Ovis aries, Capra hircus, Canis familiaris, Equus caballus, Bos taurus y Sus sp., además de identificar algunos restos de aves y peces que no serán tratados en este estudio (Pérez de Ayala 2011a y 2001b). Con relación a la fauna silvestre destaca el Cervus elaphus, Erinaceus europeus y Oryctolagus cuniculus. A las especies identificadas habría que añadir un gato que ha aparecido en conexión anatómica, pero dado que los restos de este animal se han dejado in situ (fig. 6), su análisis no se ha incluido en este trabajo.

Las unidades estratigráficas que contienen los restos óseos de macrovertebrados del yacimiento se pueden agrupar según su proceso de formación en: a) Unidades relacionadas con interfacies horizontales de ocupación (depositadas sobre pavimentos con y sin conexión anatómica) (45\% de las muestras estudiadas).

b) Vertidos residuales en las calles, originados por la falta de un proceso de eliminación de residuos. $\mathrm{La}$ acumulación de detritos provocó que de manera periódica se tuviera que repavimentar para el mantenimiento de los espacios públicos. Esto ha conllevado la conservación de numerosos restos óseos desechados tras su consumo $(11,46 \%)$.

c) Unidades estratigráficas relacionadas con transformaciones constructivas y espaciales entre periodos (explanaciones, desechos de expolios para reutilización de material constructivo, etc.) (19,72\%).

d) Relleno de elementos interfaciales verticales (vertidos en fosas) $(23,7 \%)$.

\subsection{Métodos}

El análisis que hemos realizado de los restos óseos del Teatro Cómico se ha dividido en varias secciones,

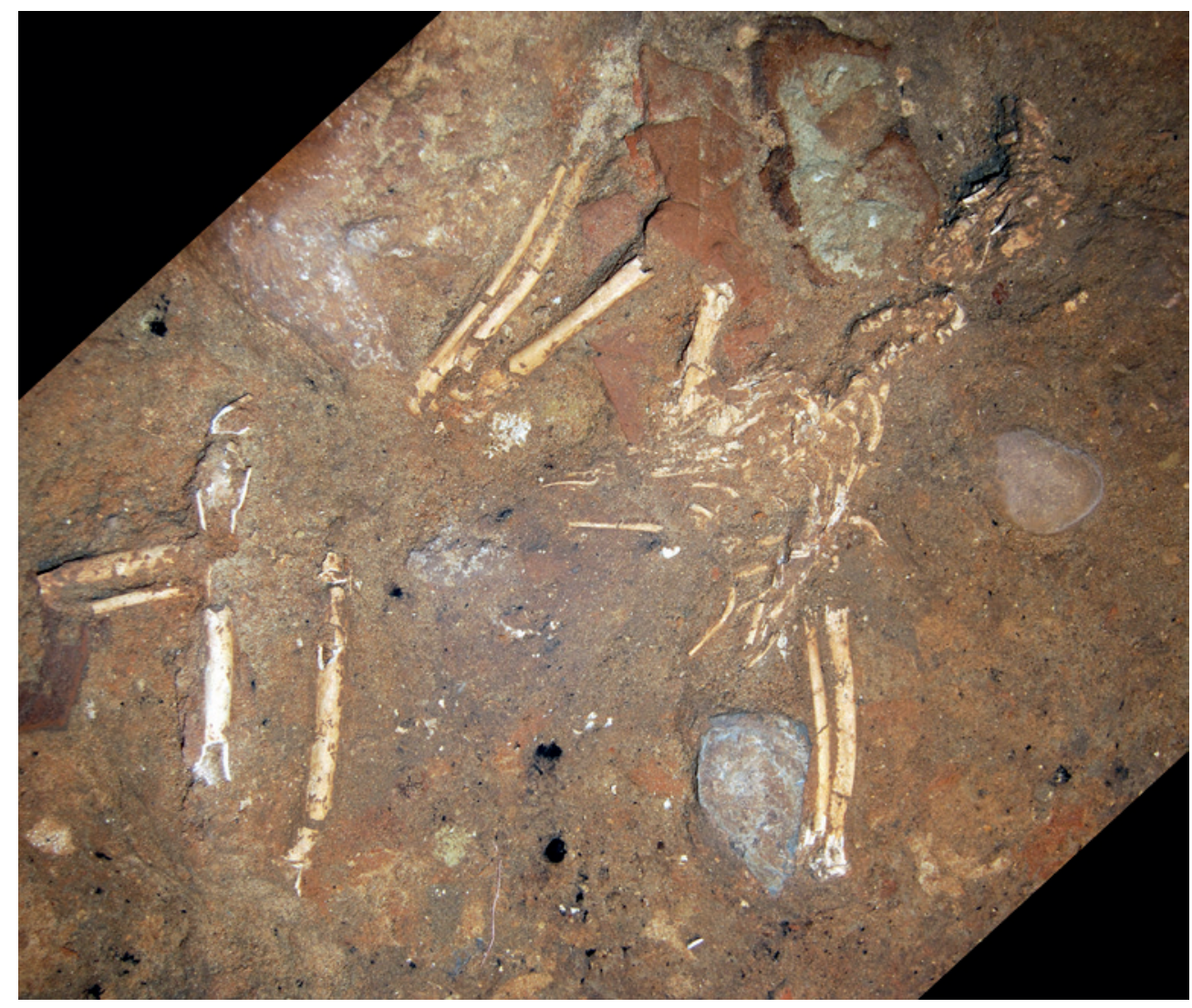

Figura 6. Felino en conexión esquelética en una de las calles (J.M.P.). 
que son: el estudio taxonómico, los patrones de mortandad y los perfiles esqueléticos.

Para realizar la identificación taxonómica se ha seguido a Lavocat (1966), Pales y Lambert (1971), Schmid (1972), Martín y Blázquez (1983) y Hilson (1992). Para casos más específicos se ha consultado a Boesseneck (1969), Payne (1985) y Prummel y Fresch (1986), y en la diferenciación entre Ovis aries, Capra hircus y Capra pirenaica a Fernández (2001). En cuanto a la determinación de Cervus elaphus y Bos taurus se ha seguido a Prumel (1988). Así mismo, junto a los trabajos de estos autores, se ha manejado material de referencia.

Para los casos de aquellos restos que no han podido identificarse taxonómicamente, pero de los que, sin embargo, sí han podido determinarse sus perfiles esqueléticos, se han agrupado en especies de distintas categorías según su talla. Así, los animales de talla grande están compuestos por vacas y caballos, los medianos por ciervo o burro, y los de talla pequeña por ovicápridos y suidos. Sólo en los casos donde no ha podido precisarse ni la especie ni el tamaño al que pertenecen, se los ha considerado indeterminables.

Con relación a la cuantificación de los restos se ha trabajado en dos aspectos; por un lado el cálculo del NR (Número de Restos) y por el otro el del MNI (Mínimo Número de Individuos), siguiéndose para este último cálculo a Brain (1969) en lugar de Binford (1978). Para los patrones de edades se han distinguido cinco grupos de edad que son: neonatos, infantiles, juveniles, adultos y seniles. Para calcular las edades se han seguido los trabajos de Pérez Ripoll (1988) y de Couturier (1962) para referirse a los ovicápridos, los de Mariezkurrena (1983), Klein et al. (1983) y Brown y Chapman (1991 a y b) para el ciervo, los de Rollet y Chiu (1994) y Bridault et al. (2002) para los suidos y los de Levine (1982) y Guadelli (1998) para los équidos. Dentro de la categoría de individuos adultos se han considerado como tales aquellos individuos que presentan una edad comprendida desde su estado adulto hasta que alcanzan el $75 \%$ de su esperanza de vida. Los seniles están comprendidos por aquellos que superan el $75 \%$ de su esperanza de vida. En cuanto a los juveniles nos referimos a los que se sitúan en torno a los 2-3 años de edad, los infantiles los menores de 2 años, y los neonatos son los recién nacidos.

En lo referente a la cuantificación del MNI, hemos computado dos tipos de MNI según se calcule de una u otra manera. De este modo, puede contarse de forma global, considerando todas las UUEE de un momento como parte de un único conjunto, o por el contrario se puede computar cada UE de forma independiente como unidades propias, sumando al final los resultados que proporciona cada UE. De esta segunda manera se tiende a incrementar el número de MNI y por consiguiente su representatividad estadística.

Para la representación anatómica cada hueso se ha incluido en una serie de secciones que son: la porción craneal comprendida por los huesos del cráneo y la mandíbula, la porción axial integrada por vértebras, costillas, escápula y pelvis siguiendo las razones aludidas por Yravedra (2006) y el esqueleto apendicular que se subdivide en elementos superiores (húmero, fémur, tibia y radio-ulna) e inferiores (metapodios y los huesos compactos).

\section{RESULTADOS}

\subsection{Estudio Zooarqueológico: Taxonomía y patrones de mortandad}

La fauna recuperada en el Teatro Cómico asciende a 1.719 restos cuya fragmentación ha dificultado la determinación, pero cuya buena conservación ha permitido determinar el 55,4\% de la muestra. Asimismo, se aprecia entre los animales determinados un espectro faunístico variado, con predominio de mamíferos y presencia de aves y peces.

Entre las especies domésticas hay ovicápridos, vacas, cerdos, perros y caballos. Entre los animales silvestres se han recuperado principalmente restos de ciervo y conejo. El grupo que ha tenido una mejor representación es el de la fauna doméstica, que comprende la mayor parte de los animales identificados en el yacimiento. De este modo la fauna silvestre sólo ha proporcionado un $1 \%$ del total de la muestra ósea identificada

Si se analiza detenidamente cada grupo se observa que entre las especies domésticas destacan los ovicápridos, incluyendo dentro de este grupo las ovejas y las cabras, con un $29,49 \%$ sobre el total de los restos y con el $53 \%$ sobre los restos determinables. Le sigue en importancia la cabaña bovina, con un 19,84 \% del total y el $35,8 \%$ de los determinables. Entre el ganado vacuno, y atendiendo a su diferenciación entre bueyes, toros y vacas, la escasez de restos con biometrías no ha permitido identificar con seguridad bueyes en el yacimiento (Estaca e Yravedra 2013). El tercer taxón en importancia es el grupo de los suidos, con un $4,65 \%$ del total de los restos y el $8 \%$ de los determinables. Tras estos tres animales, continúan el perro y el caballo, con una representación muy reducida inferior al 1\% de los restos. Con relación a los animales silvestres su representación también es muy pequeña, mostrando porcentajes mínimos (tabla 1, fig. 7). 


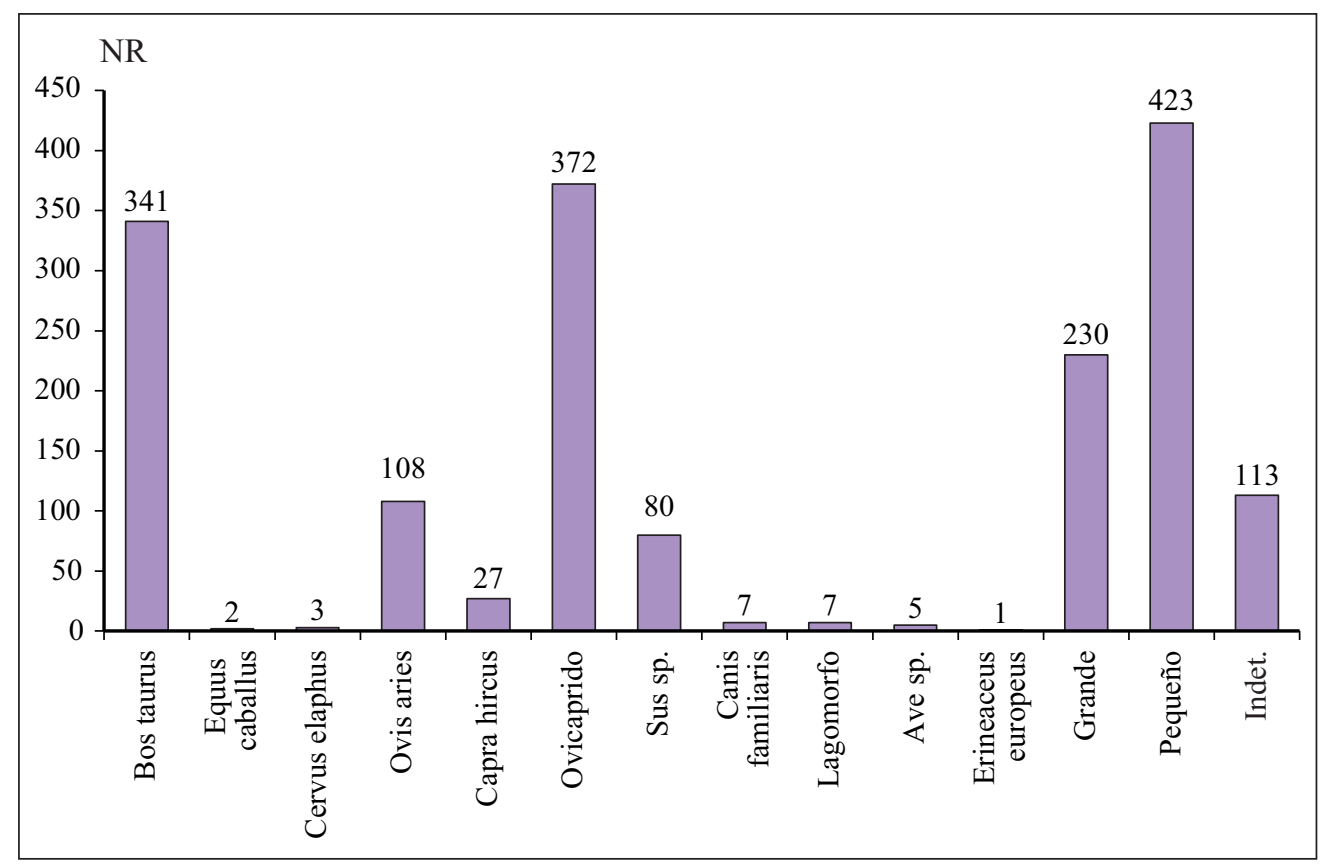

Figura 7. Perfiles

taxonómicos en Número de Restos (NR)

Tabla 1. Perfiles taxonómicos en función del NR (Número de Restos)

\begin{tabular}{|c|c|c|c|}
\hline & \multicolumn{3}{|c|}{ Teatro Cómico } \\
\hline Taxón & NR & $\%$ Total & $\begin{array}{l}\% \text { determi- } \\
\text { nables }\end{array}$ \\
\hline Bos taurus & 341 & 19,84 & 35,8 \\
\hline Equus caballus & 2 & 0,12 & 0,2 \\
\hline Cervus elaphus & 3 & 0,17 & 0,3 \\
\hline Ovis aries & 108 & 6,28 & 11,3 \\
\hline Capra hircus & 27 & 1,57 & 2,8 \\
\hline Ovis / Capra & 372 & 21,64 & 39,0 \\
\hline Sus sp. & 80 & 4,65 & 8,4 \\
\hline Canis familiaris & 7 & 0,41 & 0,7 \\
\hline Oryctolagus cuniculus & 7 & 0,41 & 0,7 \\
\hline Erinaceus europeus & 1 & 0,06 & 0,1 \\
\hline Ave sp. & 5 & 0,29 & 0,5 \\
\hline $\begin{array}{l}\text { Macromamíferos indet. } \\
\text { de talla grande }\end{array}$ & 230 & 13,38 & \\
\hline $\begin{array}{l}\text { Micromamíferos indet. } \\
\text { de talla pequeña }\end{array}$ & 423 & 24,61 & \\
\hline Indeterminados & 113 & 6,57 & \\
\hline Total & 1.719 & 100,00 & \\
\hline
\end{tabular}

Respecto al MNI (tabla 2, fig. 8), los ovicápridos mantienen su importancia al representar el $57 \%$ de los individuos. El bovino es el segundo grupo con un $15 \% \mathrm{y}$ los suidos, con un $12 \%$ el tercero, siendo las tres cabañas la triada mediterránea. Los animales peor representados son el perro y el caballo, con un bajo porcentaje con relación a los tres grupos anteriores. Por consiguiente se puede afirmar que el MNI se corresponde con el NR.

$\mathrm{Al}$ observar los porcentajes obtenidos por unidades estratigráficas (fig. 9), considerando cada unidad como un ente independiente, se aprecia el mismo esquema que en el MNI general. Por tanto, el conjunto de los ovicápridos supera el 55\% de la muestra, la cabaña bovina representa en este caso el $24 \%$, y el cerdo sigue con un $10 \%$. Hay varios animales que poseen un porcentaje muy bajo; entre ellos, el conejo muestra un $4 \%$ y el perro un 2\%. El resto de los animales que aparecen en la muestra tiene una representación total del 4,5\%.

Por tanto, se puede decir que con relación a los animales representados la muestra se acrecienta en MNI al hablar de UE (fig. 10), siendo el caso más claro la cabaña bovina, donde el número de individuos que se obtiene de manera general es de 6 , y por el contrario si vemos la representación por UE tenemos 43 individuos, incrementando la muestra en un $700 \%$. Otro caso es el de la cabaña lanar que en el apartado general presenta 23 individuos, pero al compararla con las UUEE obtiene 99, es decir, la muestra se incrementa en un $400 \%$; otros casos representativos son el cerdo y los 
Figura 8. Porcentajes de los perfiles taxonómicos en MNI (Mínimo Número de Individuos).

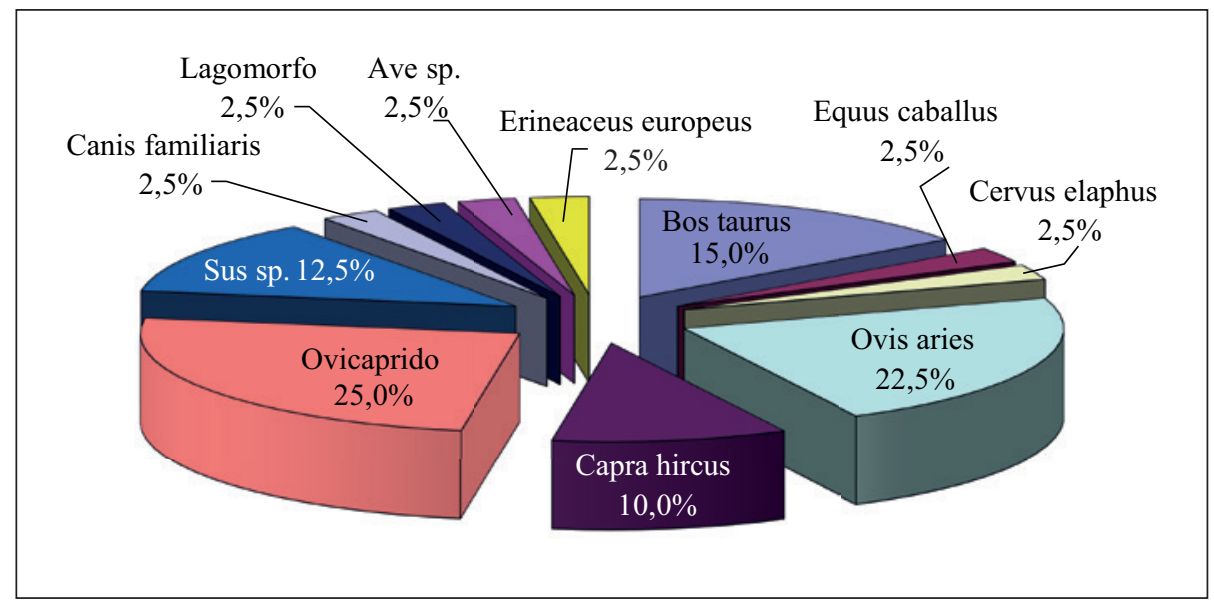

Tabla 2. Patrones de representación taxonómicos según el MNI (Mínimo Número de Individuos).

\begin{tabular}{|l|c|c|c|c|c|c|c|}
\hline \multicolumn{1}{|c|}{ Taxón } & Neonato & Infantil & Juvenil & Adulto & Senil & Total & $\%$ \\
\hline Bos taurus & 0 & 0 & 1 & 4 & 1 & 6 & 15,00 \\
\hline Equus caballus & 0 & 0 & 0 & 1 & 0 & 1 & 2,50 \\
\hline Cervus elaphus & 0 & 0 & 0 & 1 & 0 & 1 & 2,50 \\
\hline Ovis aries & 0 & 1 & 1 & 6 & 1 & 9 & 22,50 \\
\hline Capra hircus & 0 & 1 & 1 & 2 & 0 & 4 & 10,00 \\
\hline Ovicáprido & 1 & 1 & 1 & 7 & 0 & 10 & 25,00 \\
\hline Sus sp. & 0 & 1 & 1 & 2 & 1 & 5 & 12,50 \\
\hline Canis familiaris & 0 & 0 & 0 & 1 & 0 & 1 & 2,50 \\
\hline Lagomorfo & 0 & 0 & 0 & 1 & 0 & 1 & 2,50 \\
\hline Ave sp. & 0 & 0 & 0 & 1 & 0 & 1 & 2,50 \\
\hline Erineaceus europeus & 0 & 1 & 0 & 0 & 0 & 1 & 2,50 \\
\hline
\end{tabular}

Figura 9. Porcentajes de los perfiles taxonómicos en MNI (Mínimo Número de Individuos) por Unidades.

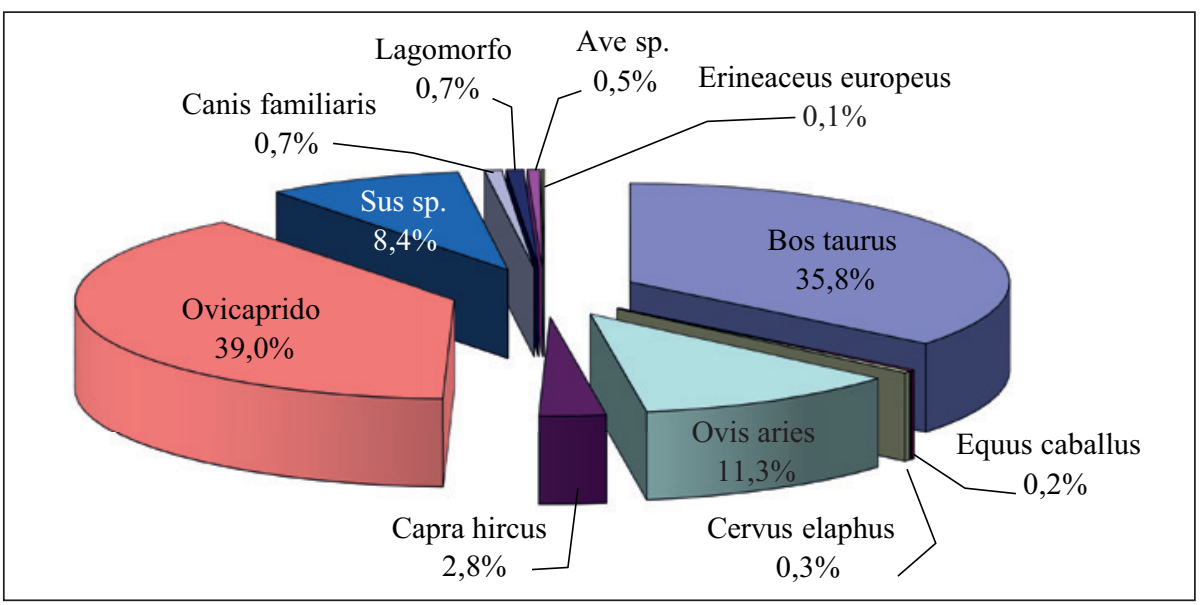




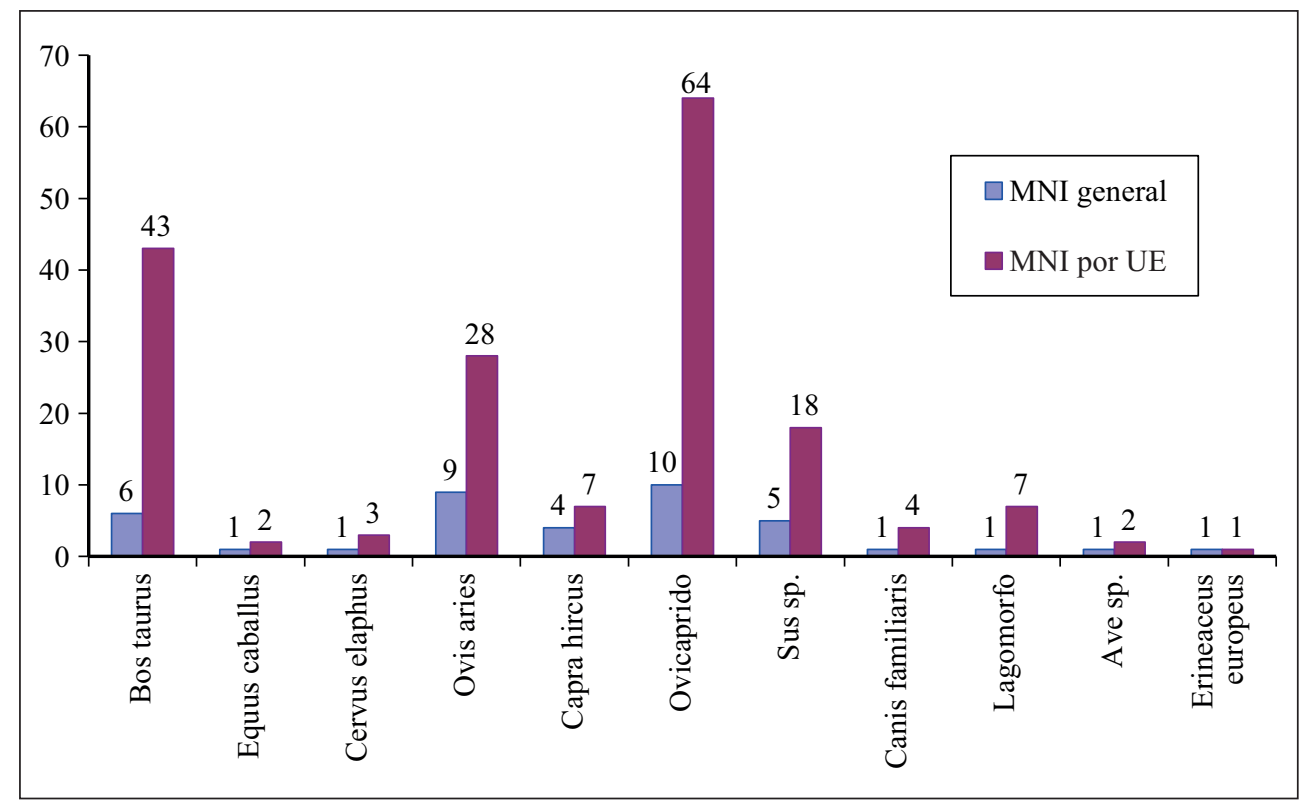

Figura 10.

Comparativa de los MNI (Mínimo Número de Individuos) total y por unidades. conejos, con un incremento de $360 \%$ y $700 \%$ respectivamente (fig. 10). El resto de la muestra no presenta grandes variaciones pues sólo tiene una diferencia entre 2 y 3 individuos. En definitiva, estos perfiles se ajustan a lo visto en el NR.

Entre los patrones de mortandad destaca el grupo de los adultos en todos los taxones, lo que sugiere que la funcionalidad de los animales no estaba tan orientada a la producción cárnica como a la productividad lanera, láctea o incluso como fuerza de trabajo o carga (tabla 2). La cultura material encontrada en el yacimiento efectivamente parece confirmar estas ideas. Aunque todavía está en proceso de estudio, se han documentado diferentes tipos de recipientes, destacando algunos fragmentos de queseras, elementos precisamente orientados a la producción de lácteos. Del mismo modo, son también abundantes los elementos textiles, entre los que predominan pesas de telar, fusayolas, agujas, etc. En cuanto a la fuerza motriz como bestia de carga, los bóvidos pudieron ayudar en la tracción de carros y teniendo en cuenta el contexto marítimo pesquero en el que nos situamos, es más que probable que se emplearan también para el acarreo del atraque de barcos y en el despesque de las jábegas de tiro desde la playa (Delgado 2008). La utilización de la fuerza del buey en las artes de pesca fue empleada en los siglos II-III d.C. por los mesios (Tracia) para la captura del siluro (Claudio Eliano, De Natura Animalium, XIV, 25). Los testimonios más antiguos en el sur peninsular son del siglo XVIII como fuerza para la pesca de arrastre (Mirabent 1850; Delgado 2008), aunque es lógico plantarse que su uso provenga -al igual que las jábegas- de tradiciones más antiguas. Sin embargo, tal consideración sólo podemos plantearla como posibilidad, ya que por el momento carecemos de patologías asociadas a este tipo de usos y tampoco se ha atestiguado biométricamente la presencia de bueyes en el yacimiento.

Entre los ovicápridos se han identificado individuos neonatos, infantiles, juveniles y adultos que hacen referencia a diferentes usos de estos animales. Así la presencia de infantiles, juveniles y adultos jóvenes sugiere que pudieron explotarse para la producción o el aprovechamiento cárnico. Por otra parte, el predominio del grupo de los adultos, con más del $50 \%$ de los individuos representados, nos indica que la principal función de estos animales en el Cómico fue la producción de lana y leche, tal y como además sugieren los elementos de cultura material antes aludidos.

Con relación al estudio de las superficies óseas, la presencia de marcas de corte en todas las cohortes de edad sugiere que todos los animales, independientemente de su edad, fueron aprovechados cárnicamente, incluidos aquellos que estaban destinados a otras funciones; sin embargo, al cesar su productividad lanera o láctea, también eran sacrificados para aprovechar su carne.

En los suidos también se han observado individuos infantiles y juveniles que podrían estar relacionados con su aprovechamiento alimenticio. Este aspecto plantea un interesante debate, dadas las implicaciones que tiene el consumo del cerdo entre las 
poblaciones semitas frente a las indígenas (Morales et al. 1995: 526-527). Sin embargo, su resolución parece complicada si consideramos la posibilidad de que entre los poblados fenicios pudiera haber poblaciones mixtas. En consecuencia, el cerdo no es un marcador significativo en la distinción de yacimientos fenicios o indígenas.

En cuanto al vacuno, también predominan los adultos, lo que podría sugerir una relación con la producción de lácteos como hemos mencionado más arriba, en función de lo que revelan los materiales cerámicos citados. La posible presencia de bueyes podría implicar funciones ligadas a la carga y la tracción. Sin embargo por el momento no hemos obtenido estimaciones biométricas que confirmen su representación en el Teatro Cómico. En cuanto a otros posibles usos, el análisis de las superficies óseas parece reflejar que, independientemente de sus funciones, todos los animales también fueron explotados cárnicamente.

\subsection{Representación taxonómica en función del NR y el MNI por periodos}

Los perfiles taxonómicos del Teatro Cómico en función de los tres periodos fenicios identificados en el yacimiento (Periodo II, III y IV) ofrecen una representación diferencial según unas fases u otras. De esta manera, el periodo II es el que ha ofrecido una mayor cantidad de restos e individuos (tablas 3 y 4, en página siguiente). Según la representación taxonómica

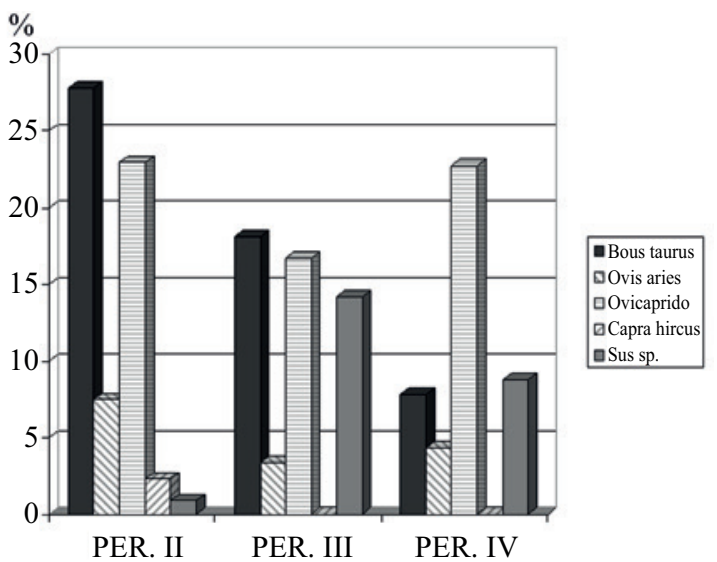

Figura 11. Representación porcentual del NR (Número de Restos) de las especies principales del Teatro Cómico por periodos. los ovicápridos son el grupo más numeroso tanto en el NR como en el MNI. Le segunda especie más importante es la vaca, lo que se ajusta a los perfiles taxonómicos globales descritos con anterioridad (tablas 1 y 2, figs. 1-5). Las demás especies apenas están representadas, incluyendo los suidos con apenas un 1\%. El periodo III muestra una representación modesta con algo más de 350 restos. Taxonómicamente, los ovicápridos siguen siendo el grupo principal, seguido de la vaca y posteriormente del cerdo, que en comparación con el periodo anterior manifiesta un incremento muy importante, con el $14,16 \%$ de los restos y el 25\% del MNI (tablas 3 y 4). En cuanto al periodo IV, su representatividad es menor, dado su escaso NR. Sin embargo, muestra una tendencia significativa al aumentar el cerdo, que se convierte en la segunda especie más importante por detrás de los ovicápridos, aunque su MNI sea similar al del periodo III.

En función de estos resultados, y analizando los taxones principales, se observan tres circunstancias que marcan la representatividad faunística del Teatro Cómico.

En primer lugar destaca la predominancia de los ovicápridos en toda la secuencias (fig. 11). A continuación, está el descenso progresivo de Bos taurus, el cual se agudiza en el periodo IV según el NR, aunque no se note tanto en el MNI (fig. 12). Al tiempo que la vaca disminuye, los suidos muestran un fuerte incremento a partir del periodo III, pasando del $0,88 \%$ al $14,16 \%$ en el NR y el $25 \%$ en el MNI, convirtiéndose en la segunda especie más importante de los periodos III y IV, al menos en el MNI.

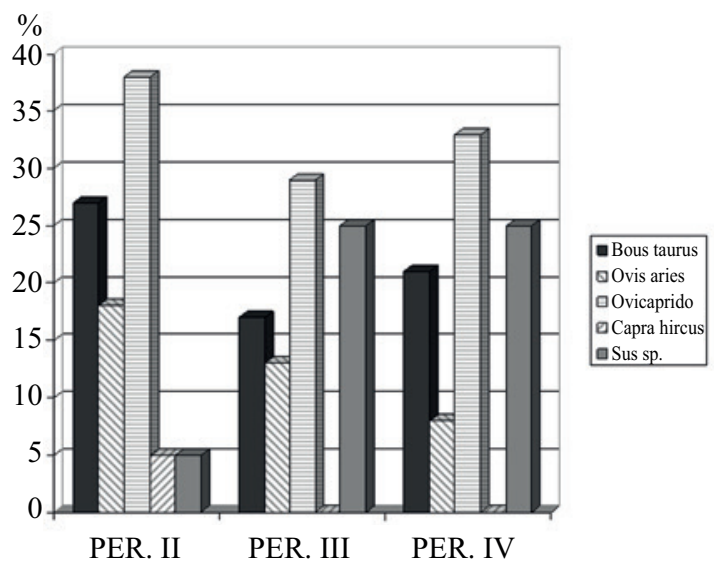

Figura 12. Representación porcentual del MNI (Mínimo Número de Individuos) de las especies principales del Teatro Cómico por periodos. 
Tabla 3. Representación Taxonómica en función del NR (Número de Restos) por periodos.

\begin{tabular}{|c|c|c|c|c|c|c|}
\hline \multirow{2}{*}{ Taxón } & \multicolumn{2}{|c|}{ Periodo II } & \multicolumn{2}{|c|}{ Periodo III } & \multicolumn{2}{|c|}{ Periodo IV } \\
\hline & NR & $\%$ & NR & $\%$ & NR & $\%$ \\
\hline Bos taurus & 259 & 22,79 & 64 & 18,13 & 18 & 7,86 \\
\hline Equus caballus & 1 & 0,08 & 1 & 0,28 & & 0,00 \\
\hline Cervus elaphus & 1 & 0,08 & 1 & 0,28 & 1 & 0,43 \\
\hline Ovis aries & 85 & 7,48 & 12 & 3,39 & 10 & 4,36 \\
\hline Ovicápridos & 261 & 22,97 & 59 & 16,71 & 52 & 22,70 \\
\hline Capra hircus & 26 & 2,28 & & 0,00 & & 0,00 \\
\hline Sus sp. & 10 & 0,88 & 50 & 14,16 & 20 & 8,73 \\
\hline Canis familiaris & 4 & 0,35 & 3 & 0,84 & & 0,00 \\
\hline Oryctolagus cuniculus & 4 & 0,35 & 1 & 0,28 & 2 & 0,87 \\
\hline Ave sp. & 5 & 0,44 & & 0,00 & & 0,00 \\
\hline Erinaceus europeus & 1 & 0,08 & & 0,00 & & 0,00 \\
\hline Grande & 127 & 11,17 & 48 & 13,59 & 55 & 25,01 \\
\hline Pequeño & 247 & 21,74 & 105 & 29,74 & 71 & 31 \\
\hline Indet. & 104 & 9,15 & 9 & 2,54 & & 0,00 \\
\hline Total & 1.136 & & 353 & & 229 & \\
\hline
\end{tabular}

Tabla 4. Representación Taxonómica en función del MNI (Mínimo Número de Individuos) por periodos.

\begin{tabular}{|l|c|c|c|c|c|c|}
\hline \multirow{2}{*}{ Taxón } & \multicolumn{2}{|c|}{ Periodo II } & \multicolumn{2}{c|}{ Periodo III } & \multicolumn{2}{c|}{ Periodo IV } \\
\cline { 2 - 7 } & MNI & $\%$ & MNI & $\%$ & \multicolumn{2}{c|}{ MNI } \\
\hline Bos taurus & 34 & 26,56 & 4 & 16,67 & 5 & 20,83 \\
\hline Equus caballus & 1 & 0,78 & 1 & 4,17 & 0 & 0,00 \\
\hline Cervus elaphus & 1 & 0,78 & 1 & 4,17 & 1 & 4,17 \\
\hline Ovis aries & 23 & 17,97 & 3 & 12,50 & 2 & 8,33 \\
\hline Ovicápridos & 49 & 38,28 & 7 & 29,17 & 8 & 33,33 \\
\hline Capra hircus & 7 & 5,47 & 0 & 0,00 & 0 & 0,00 \\
\hline Sus sp. & 6 & 4,69 & 6 & 25,00 & 6 & 25,00 \\
\hline Canis familiaris & 3 & 2,34 & 1 & 4,17 & 0 & 0,00 \\
\hline Oryctolagus cuniculus & 4 & 3,13 & 1 & 4,17 & 2 & 8,33 \\
\hline Total & 128 & & 24 & & 24 & 2 \\
\hline
\end{tabular}




\subsection{Patrones de aprovechamiento: perfiles esqueléticos}

Los perfiles esqueléticos revelan una representación de todas las secciones óseas en los ovicápridos, la vaca y los suidos, de manera que todos ellos fueron aportados de manera completa al asentamiento.

A pesar de este tipo de aporte, en la tabla 5 se observa que hay unos elementos mejor representados que otros $\mathrm{y}$, efectivamente, la porción craneal es la más abundante. Esto se puede deber a dos circunstancias: por un lado, a la gran fragmentación que suele tener el cráneo al ser un elemento fácilmente quebradizo y, por otro, a la gran cantidad de dientes frente a otros elementos óseos. Por el contrario, los elementos axiales son los huesos menos numerosos, salvo la excepción de los pertenecientes a los ovicápridos, que han alcanzado una buena representación (tabla 5). Entre los elementos apendiculares, los huesos superiores son los más importantes tanto en los ovicápridos como en la vaca. En cambio, para los suidos están muy mal representados estos elementos, al igual que los huesos axiales, lo que podría estar relacionado con la funcionalidad cárnica del cerdo tal y como se ha explicado anteriormente, confirmándose además por algunas marcas de corte identificado entre sus restos.

\section{DISCUSIÓN}

Según los datos analizados en el texto, el grupo de los ovicápridos es el más importante durante todas las épocas del yacimiento. Le sigue el ganado vacuno en el Periodo II, y el porcino en el Periodo IV. Para el periodo III, la representación del ganado vacuno y el porcino cambia en función del NR o el MNI. En cualquier caso, el grupo de los ovicápridos es el principal, y al igual que los suidos, su importancia aumenta de forma progresiva, mientras que la del vacuno se va reduciendo. Con relación a otras especies domésticas, caballos y perros también aparecen, pero su representación es poco significativa y poco podemos decir de ellos. Así, en lo que respecta al perro, no se ha podido encontrar ninguna evidencia que lo relacione con episodios de sacrificios rituales (Niveau de Villedeary y Castro 2008) que tiene que ver con el contexto funerario y ritual en el que se han encontrado evidencias de estas prácticas, por no mencionar que son de tres a cinco siglos posteriores a los contextos que aquí se están analizando. En cuanto a las especies silvestres, la funcionalidad del ciervo suele estar ligada a prácticas cinegéticas, para la que el perro podría contribuir ayudando en este tipo de tareas. Sin embargo, no hay que olvidar que el hábitat de los ciervos son ecotonos entre zonas boscosas o cubiertas con vegetación arbustiva y áreas abiertas con una producción abundante de plantas herbáceas (Carranza 2011), un ecosistema que debía diferir del de la isla de Erytheia. Por lo tanto, la aparición de los ejemplares del Teatro Cómico debe tener un origen alóctono, es decir, que debieron de ser cazados en los territorios próximos de la Península, como queda atestiguado por la aparición de este taxón en otros yacimientos, por ejemplo los fondos de cabaña de Campillo (Cáceres 1996) y Pocito Chico (Riquelme 2001). En algún caso se ha sugerido que la producción agrícola de cereal podría atraer a especies como el ciervo, que se aprovecharía del grano producido, de modo que en cierta manera el ciervo sería una especie de plaga seguidora de los cultivos humanos (Bernáldez y Bernáldez 2000), aunque esto no puede aplicarse al Cómico dada su situación topográfica y su funcionalidad, lugar mucho más dedicado al comercio y a la administración que a la producción agrícola, que en su mayor parte se obtendría del hinterland a través del importante asentamiento fenicio en tierra firme del Castillo de Doña Blanca. Haciendo alusión a esto mismo debemos reseñar además que el suelo de la isla no era apto para el cultivo de cereales.

Los patrones de mortandad han mostrado un predominio de adultos en todas las cabañas ganaderas. La escasez de marcas de cortes sugiere, junto a este predominio de adultos, que los animales no fueron especialmente explotados por su carne y que pudieron utilizarse para otros fines. Así, los ovicápridos fueron destinados principalmente a la producción de leche y a la producción de lana. Lo mismo sucede con los bovinos que parecen estar asociados a la producción de leche. Algunas de las evidencias materiales encontradas en el Cómico, como fusayolas, queseras, agujas o pesas de telar pueden confirmar este tipo de usos. Otro dato que refuerza la funcionalidad de la fauna en el Cómico, lo encontramos en el fuerte contraste que existe entre los patrones de mortandad de este sitio y los de centros ceremoniales como Montemolín, donde ovicápridos, suidos y bovinos eran sacrificados a edades tempranas, inferiores a 2 años (De la Bandera et al. 1995; Chaves et al. 2000).

Por último, en los perfiles esqueléticos de los suidos se observa un cierto sesgo de aquellas porciones anatómicas más cárnicas, los elementos apendiculares superiores delanteros y traseros. Ello podría deberse a varias 
Tabla 5. Perfiles esqueléticos en los taxones determinables.

\begin{tabular}{|c|c|c|c|c|c|c|c|c|c|}
\hline Parte anatómica & $\begin{array}{c}\text { Bos } \\
\text { taurus }\end{array}$ & $\begin{array}{c}\text { Equus } \\
\text { caballus }\end{array}$ & $\begin{array}{l}\text { Cervus } \\
\text { elaphus }\end{array}$ & $\begin{array}{l}\text { Ovis } \\
\text { aries }\end{array}$ & $\begin{array}{l}\text { Capra } \\
\text { hircus }\end{array}$ & $\begin{array}{l}\text { Ovis / } \\
\text { Capra }\end{array}$ & Sus sp. & $\begin{array}{c}\text { Canis } \\
\text { familiaris }\end{array}$ & $\begin{array}{l}\text { Oryctolagus } \\
\text { cuniculus }\end{array}$ \\
\hline Cuerno & 13 & & & & & & & & \\
\hline Cráneo & 105 & & & & & 20 & & & \\
\hline Maxilar & 4 & & & 5 & & 4 & 2 & & \\
\hline Mandíbula & 28 & & & 12 & 5 & 22 & 7 & 1 & \\
\hline Diente & & & & & & 5 & 2 & & 1 \\
\hline Incisivo & 1 & & & & & & & & \\
\hline Colmillo & & & & & & & 3 & 1 & \\
\hline Premolar & 5 & & & 19 & 11 & 14 & 7 & & \\
\hline Molar & 29 & & & 30 & 8 & 11 & 14 & & \\
\hline Vértebra & 20 & & & & & 48 & & & \\
\hline Costilla & 14 & & & & & 19 & 2 & & \\
\hline Escápula & 7 & & & 1 & & 11 & 2 & & \\
\hline Húmero & 7 & 1 & & 10 & 1 & 23 & 3 & 1 & 2 \\
\hline Radio & 5 & & & 4 & & 20 & 2 & & 1 \\
\hline Radio-Ulna & 2 & & & & & 7 & & & \\
\hline Ulna & 2 & & & & & 7 & 2 & & \\
\hline Metacarpo & 14 & & & 7 & 1 & 27 & & & \\
\hline Astrágalo & 2 & & & 5 & & 8 & 1 & & \\
\hline Rotula & & & & 1 & & & & & \\
\hline Superior & 4 & & & & & 2 & & & \\
\hline Pelvis & 3 & & & 1 & & 6 & 2 & & \\
\hline Fémur & 7 & & & 1 & & 27 & & & 1 \\
\hline Tibia & 8 & & & 7 & & 30 & 5 & 2 & 2 \\
\hline Fíbula & & & & & & & 2 & & \\
\hline Metatarso & 9 & & 1 & 3 & 1 & 18 & & & \\
\hline Tarso & 1 & & & & & & & & \\
\hline Metapodio & 5 & 1 & & & & 9 & 16 & 2 & \\
\hline Calcáneo & 1 & & & 2 & & 5 & 1 & & \\
\hline Sesamoideo & & & & & & 1 & & & \\
\hline Falange & 38 & & 2 & & & 28 & 7 & & \\
\hline Indet. & 7 & & & & & & & & \\
\hline Total & 341 & 2 & 3 & 108 & 27 & 372 & 80 & 7 & 7 \\
\hline Craneal & 185 & 0 & 0 & 66 & 24 & 76 & 35 & 2 & 1 \\
\hline Axial & 44 & 0 & 0 & 2 & 0 & 84 & 6 & 0 & 0 \\
\hline Apend. Superior & 31 & 1 & 0 & 22 & 1 & 114 & 14 & 3 & 6 \\
\hline Apend. Inferior & 74 & 1 & 3 & 18 & 2 & 98 & 25 & 2 & 0 \\
\hline
\end{tabular}


causas, como la destrucción diferencial, la actividad destructiva de carnívoros, el intercambio, el comercio o el traslado de estas partes anatómicas en forma de paletillas y jamones a otro lugar. Las evidencias de otros yacimientos podrán en el futuro aportar más información sobre este particular, pero de momento sólo podemos plantear estas hipótesis.

En comparación con otros emplazamientos, el Teatro Cómico nos ha mostrado un conjunto con menos restos que otros yacimientos como el Castillo de Doña Blanca o Toscanos (Morales et al. 1994, 1995); sin embargo sus perfiles taxonómicos no difieren de lo documentado en los otros sitios.

Los ovicápridos son el grupo mejor representado seguido del vacuno, los suidos y, ya con una representación muy pequeña los équidos, el perro, y por último, los animales salvajes, entre los que destaca el ciervo. Similar representación la encontramos en Toscanos, Castillo de Doña Blanca, Pocito Chico y Carambolo (tabla 6) (Martín Roldán 1959; Morales et al. 1994, 1995; Cáceres 1996; Riquelme 2001b; Bernáldez et al. 2010). En cuanto al Cerro del Villar y Túmulo 1, presentan una atípica sobrerrepresentación de los suidos, que contrasta con los perfiles taxonómicos de todos los demás yacimientos. Por otro lado, el Teatro Cómico tiene un conjunto poco representativo, con solo 397, y por el momento, no existen resultados sobre su MNI.

Cuando comparamos estos resultados con los obtenidos en las estaciones tartésicas estudiadas por Morales et al. (1994, 1995) y Cereijo y Patón (1989) se observa cierta similitud respecto a los yacimientos fenicios, de modo que en la mayor parte de los sitios predominan los ovicápridos. Así lo hace en Peñalosa, Puerto 29, Puerto 101, 102, 103, Setefilla, y Cerro Macareno (Estévez 1983; Cereijo y Patón 1989; Morales et al. 1994, 1995). Sin embargo en otros sitios también abunda el ganado vacuno. Así ocurre en el Cerro Macareno, con una representación muy similar a la de los ovicápridos, en Monte Berrueco o la Calle Puerto 6 de Huelva, donde la vaca es el animal más representado (Estévez 1985; Cereijo y Patón 1989).

En los suidos se observan algunos cambios respecto a los yacimientos fenicios, de modo que los suidos siempre tienen un porcentaje superior al $12 \%$ de los restos salvo la excepción de Puerto 29 con el 3\%, pero además en algunos sitios como en Tejada 1 o el Túmulo 1 de Huelva es el animal más representado (Morales et al. 1995). En contraste con estos emplazamientos tartésicos, en los fenicios, los suidos no suelen superar el 14\% de los restos, salvo la excepción del Cerro del Villar o Montemolín.
Según esto, el Teatro Cómico muestra unos datos muy interesantes, de forma que es significativa la escasa presencia de este tipo de animal en el periodo II del yacimiento en contraste con las fases posteriores. Así, en el período III parece que el descenso de los bóvidos es compensado por un incremento considerable del cerdo, convirtiéndose en la segunda especie más significativa después de los ovicápridos (tablas 3 y 4, figs. 6 y 7). Resulta interesante relacionar estos datos con los antropológicos y el estudio de ADN mitocondrial, que han mostrado unas poblaciones fenicias procedentes de Oriente en el periodo II, mientras que en momentos posteriores se han observado poblaciones mixtas de ascendencia fenicias y europeas (Palomo y Arroyo 2011). Por tanto, queda abierta una línea de investigación con el objetivo de concretar si la interacción cultural entre fenicios y poblaciones autóctonas provocó cambios sustanciales en la ganadería y en la dieta. Para nuestro caso se produciría una correlación positiva entre la reducción de suidos y el incremento de la población fenicia.

En cuanto a la representación de las faunas silvestres, se da una situación similar entre los yacimientos fenicios y tartésicos, y salvo las excepciones de Tejada y Peñalosa, Campillo y el MNI de Pocito Chico, donde el ciervo tiene el $16 \%$ de los restos, normalmente siempre tienen frecuencias inferiores al $4 \%$.

\section{CONCLUSIONES}

El Teatro Cómico ha proporcionado un conjunto óseo donde los ovicápridos son la cabaña ganadera más amplia. Los patrones de edad han mostrado un predominio de adultos en todas las épocas, lo que relacionado con una cultura material compuesta, entre otros materiales, por una amplia evidencia de fusayolas, agujas, queseras y pesas de telar hacen pensar en una funcionalidad productiva de lana y leche. Al mismo tiempo, la vaca, segunda especie en importancia, también muestra un predominio de adultos que podría hacer referencia a la productividad láctea. Por otro lado, la ausencia de datos biométricos no ha permitido identificar la presencia de buey en el Teatro Cómico, aunque no se puede desestimar la posibilidad de que este animal estuviera en el yacimiento, ya que dada la situación del Teatro Cómico podría haber tenido una funcionalidad como bestia de tiro de carros o el acarreo de barcos. Otros usos de bovinos, caprinos y ovinos también se han documentado en el Cómico. Así, se han identificado algunas marcas de corte sobre algunos individuos que 


\begin{tabular}{|c|c|c|c|c|c|c|c|c|c|c|c|c|c|c|c|c|c|c|c|}
\hline \multirow{2}{*}{$\begin{array}{l}\text { ते } \\
0 \\
0 \\
0 \\
2\end{array}$} & $\partial^{\circ}$ & & $\stackrel{0}{0}$ & $\overrightarrow{\vec{N}}$ & $\mathscr{0}_{0}^{\circ}$ & $\stackrel{0}{0}$ & $\begin{array}{l}0 \\
i\end{array}$ & $\hat{\tilde{b}}$ & $n$ & : & $\ddot{0}$ & $\stackrel{m}{i}$ & $\stackrel{\infty}{\rightarrow}$ & : & $\because$ & $\stackrel{\circ}{\sim}$ & O. & $\ddot{\sigma}^{\prime}$ & \\
\hline & ž & & - & లో & - & - & 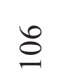 & 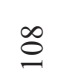 & 0 & & & $\nabla$ & $n$ & & & 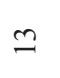 & & $\stackrel{\bullet}{\circ}$ & $\vec{I}$ \\
\hline \multirow{2}{*}{ 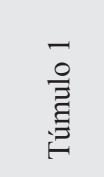 } & $\partial^{\circ}$ & & & $\stackrel{\infty}{\dot{n}^{\circ}}$ & $\therefore$ & 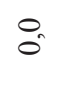 & $\stackrel{\forall}{\sim}$ & $\vec{\jmath}$ & ู̧ & & & & & & & & & & \\
\hline & 兰 & & & $\nabla$ & - & & 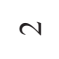 & $n$ & 0 & & & & & & & & & & $\cong$ \\
\hline \multirow{2}{*}{ 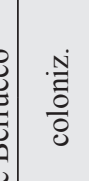 } & $\partial^{\circ}$ & & & $\frac{n}{i n}$ & & & $\begin{array}{c}0 \\
\dot{m}\end{array}$ & $\begin{array}{c}0 \\
\dot{m}\end{array}$ & in & & & & & & & & & & \\
\hline & $\tilde{z}$ & & & $\tilde{\lambda}$ & & & \pm & \pm & $N$ & & & & & - & & & & & q \\
\hline 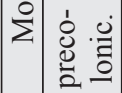 & 号 & & & \pm & & & & & & & & & & & & & & & \pm \\
\hline \multirow{2}{*}{ 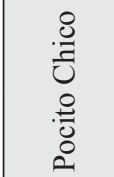 } & $\partial^{\circ}$ & & & $\underset{\sim}{0}$ & & & $\hat{\vec{m}}$ & $\hat{\vec{m}}$ & $\stackrel{i}{\tilde{\lambda}}$ & & & $\stackrel{\sim}{\alpha}$ & $\hat{\text { ते }}$ & & & $\cong$ & & $\begin{array}{l}\stackrel{\circ}{\sigma} \\
\stackrel{0}{*}\end{array}$ & 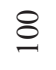 \\
\hline & 亗 & & & $\vec{F}$ & & & 8 & 8 & f & & & $\stackrel{\bullet}{-}$ & $n$ & & & $n$ & & $\infty$ & $\stackrel{ \pm}{\Xi}$ \\
\hline \multirow{2}{*}{ 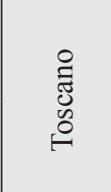 } & $0^{\circ}$ & $\begin{array}{l}\infty \\
0 \\
0\end{array}$ & & $\underset{\text { mi }}{m}$ & $\stackrel{\infty}{\stackrel{\infty}{i}}$ & $\begin{array}{l}\stackrel{0}{2} \\
\stackrel{\sim}{+}\end{array}$ & $\begin{array}{l}q \\
q \\
g\end{array}$ & $\begin{array}{l}\text { तु } \\
\text { bू }\end{array}$ & $\stackrel{\Re}{\therefore}$ & $\stackrel{\infty}{0}$ & & fo & $\begin{array}{l}n \\
\text { ñ } \\
0\end{array}$ & $\begin{array}{l}\tilde{o}_{n} \\
\tilde{o}^{\prime}\end{array}$ & & $\cong$ & $\begin{array}{l}\infty \\
0 \\
0\end{array}$ & $\stackrel{\vec{i}}{\stackrel{i}{i}}$ & 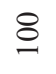 \\
\hline & $\tilde{z}$ & $m$ & & $\stackrel{\infty}{=}$ & के & $\stackrel{R}{n}$ & $\stackrel{8}{2}$ & $\underset{i}{\vec{j}}$ & $\stackrel{尺}{\sim}$ & $n$ & & $=$ & $a$ & - & & $R$ & $n$ & $\infty$ & $\begin{array}{l}\infty \\
\infty \\
n \\
n\end{array}$ \\
\hline \multirow{2}{*}{ 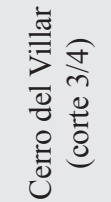 } & $\partial^{\circ}$ & & & 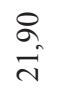 & $\stackrel{2}{=}$ & $\begin{array}{l}\text { ò } \\
\text { i }\end{array}$ & ๙ै. & 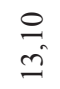 & $\begin{array}{l}\text { R } \\
\text { î }\end{array}$ & & & $\begin{array}{l}n \\
n \\
0\end{array}$ & $\underset{\sigma}{\sigma}$ & & $\begin{array}{l}n \\
n \\
0\end{array}$ & $\begin{array}{l}n \\
n \\
0\end{array}$ & in & $\underset{\text { ò }}{i}$ & 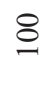 \\
\hline & $\tilde{z}$ & & & $\hat{\infty}$ & $r$ & $\infty$ & $\hat{n}$ & $\approx$ & 궈 & & & $N$ & ナ & & $\sim$ & - & - & $\infty$ & $\hat{\text { mे }}$ \\
\hline \multirow{2}{*}{ 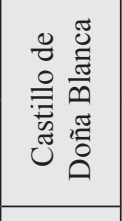 } & $0^{\circ}$ & & $\stackrel{0}{0}$ & $\stackrel{尺}{=}$ & $\begin{array}{l}\infty \\
i \\
i\end{array}$ & $\stackrel{+}{\infty}$ & $\begin{array}{l}8 \\
5\end{array}$ & $\begin{array}{l}0 \\
i \\
i\end{array}$ & $\stackrel{R}{\stackrel{R}{+}}$ & & & $\stackrel{\infty}{\stackrel{\infty}{0}}$ & $\stackrel{\infty}{\stackrel{\infty}{0}}$ & & & $\begin{array}{l}\text { तิ } \\
\text { อิ }\end{array}$ & $\stackrel{\hat{s}}{0}$ & $\stackrel{n}{=}$ & 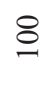 \\
\hline & 光 & & - & 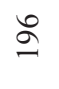 & f & $\bar{m}$ & $\stackrel{0}{=}$ & $\stackrel{n}{\stackrel{n}{I}}$ & 2 & & & $\infty$ & $\infty$ & & & 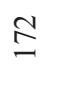 & 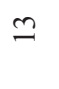 & $\tilde{\sigma}$ & $\underset{\sim}{\vec{\infty}}$ \\
\hline \multirow{2}{*}{ 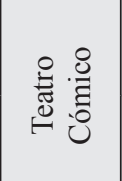 } & $0^{\circ}$ & $\stackrel{1}{\stackrel{0}{0}}$ & & $\begin{array}{l}\infty \\
\infty \\
m\end{array}$ & $\stackrel{\stackrel{\rho}{m}}{=}$ & $\begin{array}{l}+ \\
\infty \\
i\end{array}$ & $\frac{\circ}{\dot{m}}$ & $\begin{array}{l}\text { m } \\
\hat{n}\end{array}$ & & & $\underset{\infty}{\stackrel{q}{+}}$ & $\frac{t}{0}$ & $\begin{array}{l}\text { तै } \\
0 \\
0\end{array}$ & & & $\frac{1}{0}$ & $\hat{n}$ & $\stackrel{\infty}{\sim}$ & 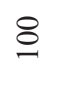 \\
\hline & 光 & $\sim$ & & $\vec{F}$ & 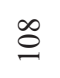 & $\hat{\imath}$ & $\underset{m}{\mathbb{N}}$ & î & & & $\infty$ & $r$ & $n$ & & & $r$ & in & $\cong$ & $\tilde{\alpha}$ \\
\hline 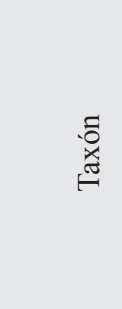 & & 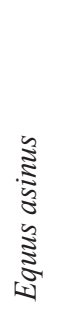 & 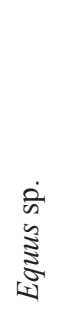 & 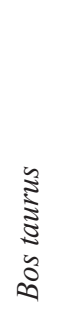 & 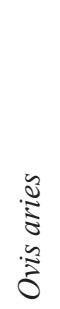 & 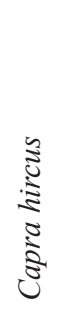 & 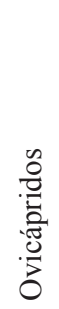 & $\begin{array}{l}\bar{\pi} \\
0 \\
0 \\
0 \\
0 \\
0 \\
0 \\
0 \\
0 \\
0 \\
0 \\
0\end{array}$ & 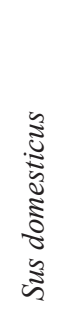 & 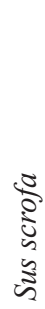 & $\begin{array}{l}\dot{2} \\
\text { के } \\
\text { जे }\end{array}$ & 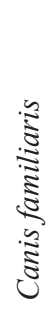 & 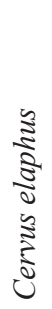 & 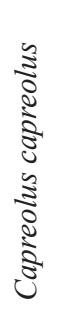 & 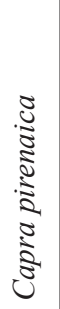 & 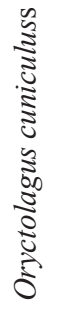 & $\begin{array}{l}\dot{\hat{n}} \\
\dot{0} \\
\dot{z}\end{array}$ & 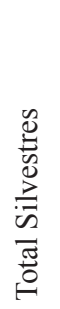 & 吾 \\
\hline
\end{tabular}




\begin{tabular}{|c|c|c|c|c|c|c|c|c|c|c|c|c|c|c|c|c|c|c|c|}
\hline \multirow{2}{*}{ 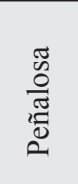 } & $a^{\circ}$ & & oे & $\hat{=}$ & $\stackrel{\sim}{=}$ & & है & $\frac{\vec{m}}{m}$ & $\begin{array}{l}\stackrel{n}{m} \\
m\end{array}$ & & & & $\exists$ & & & & & & \\
\hline & 号 & & $n$ & f & $\nabla$ & 0 & $\stackrel{\varrho}{\varrho}$ & $\hat{o}$ & fo & & & & $\underset{m}{\infty}$ & & & & & $\underset{m}{\infty}$ & $\vec{f}$ \\
\hline \multirow{2}{*}{ 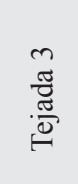 } & $a^{\circ}$ & & & $\begin{array}{l}\stackrel{0}{\sim} \\
\stackrel{\sim}{n}\end{array}$ & $\hat{\text { iे }}$ & & $\hat{\AA}$ & $\stackrel{+}{\vec{\sigma}}$ & $\begin{array}{l}\stackrel{0}{\sim} \\
\stackrel{\sim}{N}\end{array}$ & & & & $\stackrel{\sim}{\sim}$ & & & & & $\stackrel{+}{m}$ & \\
\hline & 孚 & & & $\infty$ & 6 & & 6 & $\simeq$ & $\infty$ & & & & - & & & & & - & ते \\
\hline \multirow{2}{*}{ 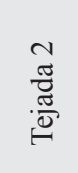 } & $a^{\circ}$ & & & ㄱ. & $\nabla$ & & f & F & $\simeq$ & & & & $\stackrel{J}{\sim}$ & & & & & $\stackrel{\Delta}{ }$ & \\
\hline & 号 & & & in & - & & 으 & $=$ & $m$ & & & & 0 & & & & & 0 & ปै \\
\hline \multirow{2}{*}{ 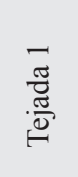 } & $a^{\circ}$ & & & $\begin{array}{l}\infty \\
n \\
n\end{array}$ & & & $\overrightarrow{\text { do }}$ & $\overrightarrow{d_{0}^{N}}$ & $\begin{array}{l}0 \\
\infty \\
\infty \\
\infty\end{array}$ & & & & $\stackrel{n}{=}$ & & & & & $\stackrel{n}{=}$ & \\
\hline & 号 & & & $a$ & & & $\stackrel{\bullet}{-}$ & $\stackrel{\bullet}{0}$ & ה & & & & 우 & & & & & 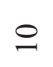 & in \\
\hline \multirow{2}{*}{ 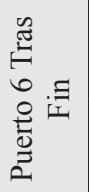 } & $a^{\circ}$ & & $\stackrel{\infty}{\infty}$ & $\begin{array}{l}n \\
\infty \\
n \\
n\end{array}$ & $\hat{r}$ & $\stackrel{?}{\rightarrow}$ & $\overrightarrow{\text { si }}$ & $\begin{array}{l}0 \\
F\end{array}$ & $\stackrel{n}{=}$ & & & $\stackrel{\infty}{\sim}$ & $\stackrel{m}{\rightarrow}$ & & & & O & $\stackrel{m}{\rightarrow}$ & \\
\hline & 孚 & & $n$ & in & 0 & - & $\stackrel{\sim}{\sim}$ & $\pi$ & $a$ & & & m & - & & & & & - & $\stackrel{\infty}{\sim}$ \\
\hline \multirow{2}{*}{ 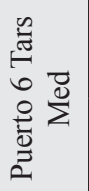 } & $a^{\circ}$ & & $\hat{0}$ & $\begin{array}{l}0 \\
\dot{q}\end{array}$ & $\overbrace{0}^{+}$ & $\vec{i}$ & $\overline{\mathrm{I}}$ & $\begin{array}{l}0 \\
\stackrel{i}{1}\end{array}$ & $\begin{array}{l}0 \\
\hat{i}\end{array}$ & & & $\stackrel{m}{F}$ & $\underset{i}{\infty}$ & & & & & $\stackrel{\infty}{i}$ & \\
\hline & 孚 & & - & 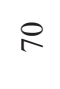 & $a$ & $n$ & 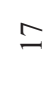 & নे & $\vec{m}$ & & & 6 & ナ & & & & & $\nabla$ & 正 \\
\hline \multirow{2}{*}{ 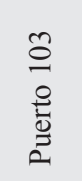 } & $a^{\circ}$ & & & ț & $\stackrel{\circ}{+}$ & $\stackrel{\Delta}{\sim}$ & \begin{tabular}{l}
0 \\
o. \\
\multirow{2}{*}{}
\end{tabular} & $\stackrel{\vec{n}}{\tilde{n}}$ & $\begin{array}{l}\infty \\
\text { I }\end{array}$ & & & & & & & & & & \\
\hline & 号 & & & $\hat{\alpha}$ & $\Xi$ & in & o & $\stackrel{\infty}{\infty}$ & in & & & 0 & ల & & & & & ల & in \\
\hline \multirow{2}{*}{ 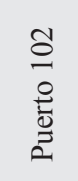 } & $a^{\circ}$ & & & $\overrightarrow{\vec{\lambda}}$ & $\stackrel{+}{\leftrightarrows}$ & $\stackrel{0}{0}$ & $\hat{n}$ & $\hat{\tilde{b}}$ & $\begin{array}{l}\stackrel{\circ}{ \pm} \\
\text { the }\end{array}$ & & & & & & & & & & \\
\hline & 号 & 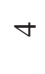 & & $\cong$ & $\infty$ & $a$ & $\underset{ల}{~}$ & $\stackrel{\sim}{\sim}$ & $\triangleright$ & & & 6 & 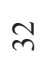 & & & & & तె & in \\
\hline \multirow{2}{*}{$\begin{array}{l}\overline{0} \\
0 \\
0 \\
0 \\
0 \\
0\end{array}$} & $a^{\circ}$ & & & $\stackrel{n}{\simeq}$ & ?ె. & 8 & $\vec{n}$ & 䒛 & $\vec{\infty}$ & & & & & & & & & & \\
\hline & 卢 & & & $\nabla$ & $\sim$ & 0 & $=$ & 2 & $a$ & & & 0 & & & & & & & $\approx$ \\
\hline \multicolumn{2}{|c|}{$\begin{array}{l}\text { 芯 } \\
\stackrel{\varpi}{\varpi}\end{array}$} & 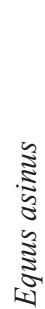 & 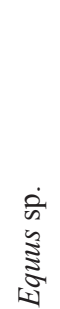 & 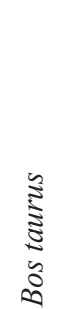 & $\begin{array}{l}\tilde{y} \\
\tilde{\Xi} \\
\tilde{z} \\
\tilde{z}\end{array}$ & 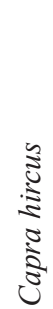 & 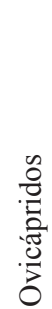 & 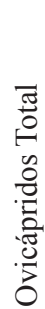 & 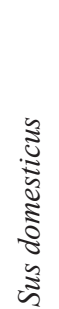 & 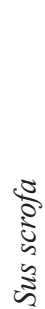 & $\begin{array}{c}\dot{n} \\
\vdots \\
\vdots \\
\tilde{n}\end{array}$ & 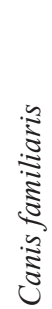 & 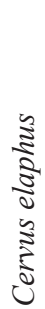 & 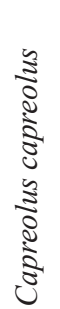 & 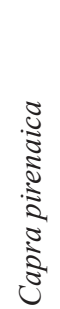 & 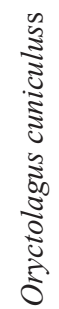 & $\begin{array}{l}\dot{2} \\
\dot{n} \\
\dot{\partial}\end{array}$ & 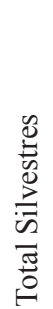 & 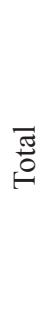 \\
\hline
\end{tabular}




\begin{tabular}{|c|c|c|c|c|c|c|c|c|c|c|c|c|c|c|c|c|c|c|c|}
\hline \multirow{2}{*}{ 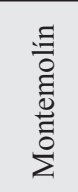 } & $\partial^{\circ}$ & & & $\vec{m}$ & $0_{0}^{\circ}$ & $\because$ & $\begin{array}{l}0 \\
\ddot{\lambda}\end{array}$ & $\begin{array}{l}0 \\
\ddot{\lambda}\end{array}$ & $\stackrel{m}{m}$ & & & & & & & & & & \\
\hline & $\tilde{z}$ & & & $\approx$ & & & $\infty$ & 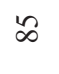 & $\hat{o}$ & & & & & & & & & & 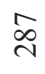 \\
\hline \multirow{2}{*}{ 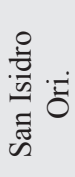 } & $\partial^{\circ}$ & & & $\stackrel{\forall}{\check{n}}$ & 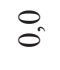 & : & $\vec{f}$ & $\bar{f}$ & $\hat{\tilde{N}}$ & & & & & & & & & & \\
\hline & 弚 & & & 0 & & & $\ddot{n}$ & $\ddot{\sim}$ & $\infty$ & & & & & & & & & & ले \\
\hline \multirow{2}{*}{ 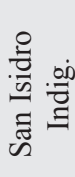 } & $0^{\circ}$ & & & $\begin{array}{l}\stackrel{\circ}{f} \\
\stackrel{f}{*}\end{array}$ & $\begin{array}{l}n \\
\tilde{n}\end{array}$ & $\stackrel{\vec{v}}{\mathrm{~d}}$ & $\stackrel{0}{\circ}$ & $\stackrel{a}{\check{z}}$ & $\stackrel{\infty}{\gtrless}$ & $\because$ & $\because$ & $\stackrel{0}{\circ}$ & $\hat{m}$ & & & & & & \\
\hline & 弚 & & & $\hat{m}$ & $\underline{m}$ & $N$ & & 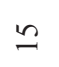 & $\nabla$ & & & & $\stackrel{\infty}{\sim}$ & & & & & & 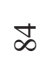 \\
\hline \multirow{2}{*}{ 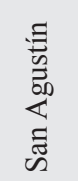 } & $0^{\circ}$ & & 乞ี & $\stackrel{0}{0}$ & $\vec{a}$ & $\begin{array}{l}\stackrel{f}{f} \\
\text { fis }\end{array}$ & $\therefore$ & $\frac{n}{n}$ & $\begin{array}{l}\text { m. } \\
\text { हे }\end{array}$ & $\therefore$ & $\because$ & : & in & $\vec{a}$ & & & & & \\
\hline & 兑 & & $n$ & & $m$ & \pm & & $=$ & 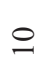 & & & & - & $n$ & & & & & $\tilde{m}$ \\
\hline \multirow{2}{*}{$\begin{array}{l}\text { 응 } \\
\text { है } \\
\text { Uేँ }\end{array}$} & $\partial^{\circ}$ & 3 & & $\stackrel{\leftrightarrow}{m}$ & $\therefore$ & $\because$ & tro & tro & $\stackrel{\stackrel{\sim}{ \pm}}{ }$ & $\because$ & : & ô & $\because$ & $\vec{m}$ & $\because$ & $\begin{array}{l}0 \\
i\end{array}$ & $\because$ & $\hat{n}$ & \\
\hline & 兑 & - & & $\cong$ & & & $\underset{\sim}{\infty}$ & $\underset{\sim}{\infty}$ & $\widetilde{\infty}$ & & & - & & $\stackrel{\infty}{\longrightarrow}$ & & $\because$ & & $m$ & $\stackrel{\infty}{i}$ \\
\hline \multirow{2}{*}{ 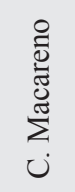 } & $\partial^{\circ}$ & & on & $\begin{array}{l}\stackrel{0}{f} \\
\vec{f}\end{array}$ & $\overrightarrow{\hat{i}}$ & $\Rightarrow$ & ले & $\begin{array}{c}m \\
\dot{q}\end{array}$ & 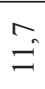 & $\because$ & $\because$ & $\exists$ & $\stackrel{0}{0}$ & $\stackrel{t}{0}$ & : & $\stackrel{b}{i}$ & 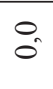 & $\hat{\imath}$ & \\
\hline & ž & & - & $\ddot{\approx}$ & $\stackrel{0}{-}$ & 0 & $\stackrel{n}{\sim}$ & $\overline{\widetilde{N}}$ & t & & & 0 & & $\sim$ & & $\Xi$ & & $\stackrel{0}{0}$ & $\underset{i}{\stackrel{g}{n}}$ \\
\hline \multirow{2}{*}{$\begin{array}{l}\frac{\pi}{\bar{E}} \\
\frac{\bar{v}}{0} \\
\tilde{\omega}\end{array}$} & $\partial^{\circ}$ & & $\stackrel{m}{\stackrel{n}{d}}$ & $\stackrel{+}{\leftrightarrows}$ & $\stackrel{n}{n}$ & $\hat{i}$ & $\hat{\vec{\lambda}}$ & $\stackrel{\sim}{\tilde{q}}$ & î & $\because$ & : & $\because$ & $\stackrel{+}{\rightarrow}$ & $\because$ & : & $\stackrel{+}{\rightarrow}$ & $\therefore$ & $\hat{i}$ & \\
\hline & $\frac{a}{z}$ & & $\stackrel{\infty}{-}$ & - & $\circ$ & N & त & $\tilde{n}$ & $\stackrel{2}{ }$ & & & & - & & & - & & $N$ & i \\
\hline \multirow{2}{*}{ 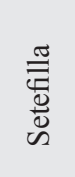 } & $\partial^{\circ}$ & & $\overrightarrow{\tilde{N}}$ & $\stackrel{\infty}{0}$ & $\stackrel{\vec{n}}{i}$ & $\vec{m}$ & के & $\stackrel{+}{q}$ & $\stackrel{\circ}{\stackrel{+}{\sim}}$ & $\therefore$ & 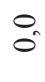 & $\stackrel{0}{\circ}$ & ले & 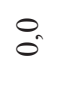 & $\therefore$ & $\hat{0}^{\infty}$ & $\therefore$ & $\stackrel{\sim}{*}$ & \\
\hline & 乥 & & 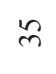 & - & $r$ & $\nabla$ & $\dddot{q}$ & in & $\vec{m}$ & & & & $n$ & & & - & & 0 & సे \\
\hline \multirow{2}{*}{ 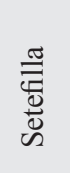 } & $\partial^{\circ}$ & & ò & से & & $\hat{0}$ & से & $\begin{array}{l}\infty \\
\dot{m}\end{array}$ & $\begin{array}{c}\text { ma } \\
\text { in }\end{array}$ & & & & & & & & & & \\
\hline & 兑 & & - & $\bar{n}$ & & - & $\hat{m}$ & $\underset{n}{\infty}$ & in & & & & & & & & & & 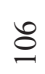 \\
\hline & 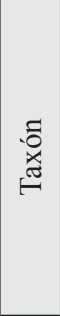 & 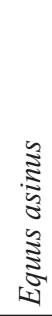 & 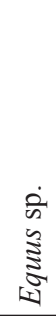 & 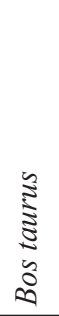 & 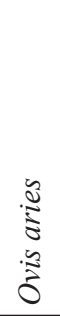 & 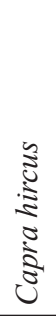 & 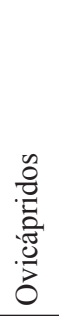 & 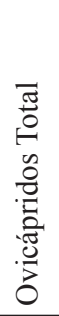 & 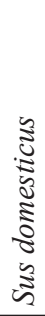 & $\begin{array}{l}0 \\
0 \\
0 \\
5 \\
5 \\
5 \\
5\end{array}$ & $\begin{array}{l}\dot{2} \\
\hat{n} \\
\hat{n}\end{array}$ & 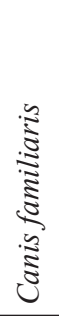 & 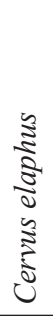 & 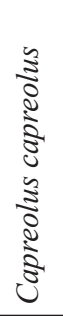 & 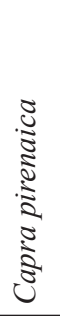 & 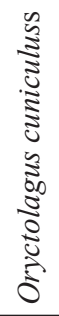 & $\begin{array}{l}\dot{8} \\
\dot{0} \\
\dot{2}\end{array}$ & 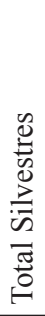 & 吾 \\
\hline
\end{tabular}


indican que los animales una vez cumplidos sus servicios en vida, se aprovecharon cárnicamente. Respecto a los suidos, tercera especie más importante, destaca que su representación cambia en función de unos momentos u otros. Así, en el periodo II se reduce su proporción justo cuando hubo una mayor población procedente de Fenicia en el yacimiento y cuyas pautas culturales son aún las mismas de la metrópolis. De momento, esta curiosidad es una observación importante que debemos contrastar en otros lugares, ya que puede ayudarnos a discernir ocupaciones semitas o tartésicas y, en consecuencia, podría ser un aspecto cultural más que nos permitiera discriminar unas y otras ocupaciones.

Los datos obtenidos permiten ampliar la cantidad de datos zooarqueológicos disponibles para este periodo. Sin embargo, aún son necesarios más análisis de este tipo que permitan contrastar la información proporcionada por el Teatro Cómico y los demás sitios citados en el texto. Todos ellos han ofrecido datos importantes sobre la ganadería de los pobladores tartésicos y fenicios del suroeste de la península ibérica, pero, en comparación con el número de lugares conocidos, su cantidad sigue siendo escasa. Sólo un estudio más profundo de un mayor número de conjuntos permitirá obtener conclusiones más precisas sobre la economía ganadera. Del mismo modo, su aplicación a diferentes tipos de ámbitos, como poblados, centros ceremoniales y necrópolis, podrá permitir ampliar las informaciones disponibles cuantitativamente y cualitativamente. Por ejemplo, contrastando los datos de distintas clases de emplazamientos se podría observar si hay diferencias taxonómicas en función de los sitios, si los patrones de mortandad cambian, o incluso si las pautas de sacrificio y aprovechamiento animal difieren de unos asentamientos a otros. En nuestro caso la predominancia de adultos en ovicápridos y bovinos hace referencia a los fines productivos de lácteos y textiles en contraposición a espacios ceremoniales como Montemolín con predominancia de individuos infantiles-juveniles que serían sacrificados en actos rituales y procesados después.

Futuros trabajos nos permitirán contrastar mejor estas cuestiones y otras más específicas, pero antes se deben estudiar más sitios para obtener informaciones que proporcionen una cobertura argumental apropiada.

\section{Agradecimientos}

Agradecemos la realización de este artículo a los revisores anónimos que han evaluado y discutido este trabajo, ya que su aportación ha sido clave para la mejoría de la exposición del estudio, además de ayudar a completar la contextualización referencial de los yacimientos fenicios del sur de la península ibérica. Agradecemos también el trabajo al CAI de Arqueometría y Análisis Arqueológico de la Universidad Complutense de Madrid y al proyecto arqueológico del Teatro Cómico del Excmo. Ayuntamiento de Cádiz. Por último queremos expresar nuestro agradecimiento al secretario de la revista por toda la paciencia que ha tenido en las diferentes revisiones del trabajo y comentar que los autores son los responsables de los datos que aquí se presentan.

\section{BIBLIOGRAFÍA}

Bernáldez, E. (1988): "Estudio faunístico”, en J. Campos Carrasco, M. Vera Reina y M. T. Moreno Menayo, Protohistoria de la ciudad de Sevilla. El corte estratigráfico San Isidoro 85-6 (Monografías de Arqueología Andaluza 1): 103-121. Sevilla, Junta de Andalucía.

Bernáldez, E. (1990): "Estudio faunístico de la excavación de San Agustín-86 en la ciudad de Málaga”, en A. Recio Ruiz (ed.), La cerámica fenicio-púnica, griega y etrusca del sondeo de San Agustín (Málaga). Monografías: 167-173. Málaga, Diputación Provincial de Málaga.

Bernáldez, E. (1997): "Estudio tafonómico y arqueozoológico", en M. Belén Deamos, Arqueología en Carmona (Sevilla). Excavaciones en la Casa-Palacio del Marqués de Saltillo: 247-262. Sevilla, Junta de Andalucía.

Bernáldez, E. y Bernáldez, M. (2000): "La basura orgánica de Lebrija en otros tiempos. Estudio paleobiológico y tafonómico del yacimiento arqueológico de la calle Alcazaba de Lebrija (Sevilla)", Boletín del Instituto Andaluz del Patrimonio Histórico 32: 134-150.

Bernáldez, E.; García-Viñas, E.; Ontiveros, E.; Gómez, A. y Ocaña, A. (2010): "Del mar al basurero: una historia de costumbres", en M.L. de la Bandera y E. Ferrer (eds.), El Carambolo. 50 años de un tesoro: 345-379. Sevilla, Universidad de Sevilla.

Binford, L.R. (1978): Nunamiut Ethnoarchaeology. Nueva York, Michigan University, Academic Press.

Boessneck, J. (1969): “Osteological Differences between Sheep (Ovis aries aries Linné) and Goats (Capra hircus hircus linné)", en D. Brothwell y E. Higgs (eds.): Sciencie in Archaeology: 331-358. Londres, Thames y Hudson.

Brain, C.K. (1969): "The contribution of Namib desert Hottentot to understanding of Australopithecus 
bone accumulations". Scientific Papers in Namibian Desert Research Station 32: 1-11.

Brown, W.A.B. y Chapman, N.G. (1991a): "The dentition of red deer (Cervus elaphus): a scoring scheme to assess age from wear of the permanent molariform teeth". Journal of Zoological London 224: 519-536.

Brown, W.A.B. y Chapman, N.G. (1991b): "Age assessment of red deer (Cervus elaphus): from a scoring scheme based on radiographs of developing permanent molariform teeth". Journal of Zoological London 225: 85-97.

Cáceres, I. (1996): “Addenda 1. Los restos de animales del yacimiento Arroyo Campillo”, en J.J. López Amador, J.A. Ruiz Gil, P. Bueno, M. de Prada (eds.), Tartesios y fenicios en Campillo, El Puerto de Santa María, Cádiz: una aportación a la cronología del Bronce final en el occidente de Europa: 157-163. Cádiz, Francisco Giles Pacheco.

Calero, M.; Bueno, A.; Pajuelo, J.M.; Navarro, M.A. y Gener, J.M a (2012): "Estudio paleopatológico fenicio mediante tomografía axial computerizada tridimensional". Paleopatología 10: 1-7.

Calero, M; Bueno, A.; Gener, J.Mª Navarro, M.A. y Pajuelo, J.M. (e.p.): "Nuevo método de estudio de restos humanos antiguos esqueletizados mediante Tomografia Axial Computerizada Tridimensional (TAC 3D) previa consolidación in situ", en A.M Niveau de Villedary (ed.), Nuevas perspectivas de investigación en Arqueología funeraria, Collezione di Studi Fenici. Roma, ISCIMA.

Carranza, A. (2011): "Ciervo-Cervus elaphus", en A. Salvador y J. Cassinello (coords), Enciclopedia Virtual de los Vertebrados Españoles. Museo Nacional de Ciencias Naturales. http://www.vertebradosibericos.org/mamiferos/pdf/cerela.pdf

Cereijo, M.A. y Patón, D. (1989): "Estudio sobre la fauna de vertebrados recuperada en el yacimiento tartésico de la calle del Puerto 6 (Huelva). Primera parte mamíferos". Huelva Arqueológica 10-11: 215-244.

Chaves, F., De la Bandera, M.L., Ferrer, E. y Bernáldez, E. (2000): "EL Complejo Sacrifical de Montemolín”, en M. E. Aubet y M. Barthélemy (eds.), Actas del IV Congreso Internacional de Estudios Fenicios y Púnicos. Volumen II: 573-582. Cádiz, Universidad de Cádiz.

Coutourier, M. A. J. (1962): Le bouquetin des Alpes. Grenoble, Arthaud.

De la Bandera, M.L.; Chaves, F. y Ferrer, E. (1999): "Ganado, sacrificio y manipulación de carnes. Una propuesta aplicada al periodo orientalizante", en P. Bueno y R. de Balbín (coords.), II Congreso de
Arqueología Peninsular: 213-219. Alcalá de Henares, Universidad de Alcalá de Henares.

De la Bandera, M.L.; Chaves, F.; Ferrer, E. y Bernáldez, E. (1995): "El yacimiento tartésico de Montemolín”, en Tartessos 25 años después. 1968-1993. Jerez de la Frontera. Publicación Conmemorativa del V Symposium Internacional de Prehistoria Peninsular: 315-332. Jerez de la Frontera, Ayuntamiento de Jerez de la Frontera.

Delgado Domínguez, A. (2008): Pesca y producción de conservas de pescado en época antigua: El litoral onubense. Estado de la cuestión (s. VI a.C.-IV d.C.). UMI Microfilmn 32926998 , ProQuestInformationandLeaming Company. https:/www.academia.edu/3195149/ DELGADO_DOMINGUEZ_A._2008_PESCA Y PRODUCCION DE CONSERVAS DE PESCADO EN EPOCA ANTIGUA EL LITORAL_ONUBENSE._ESTADO_DE_LA_ CUESTION_s._VI_a._C._-_IV_d._C._._ProQuest_ UMI_Number_3292698

Domínguez-Bella, S. (2011): "Reconstrucción del marco geológico de la Bahía de Cádiz: recursos líticos y materias primas", en J.C. Domínguez Pérez, Gadir y el Círculo del Estrecho revisados. Propuestas de la arqueología desde un enfoque social: 5973. Cádiz, Servicio de Publicaciones de la Universidad de Cádiz.

Escacena, J.L. (1985): “Gadir”. Aular 3: 39-58.

Elayi, J. (1980): "Remarques sur un type de mur phenicien". Rivista di Studi Fenici 8/2: 165-180.

Elayi, J. (1996): "Nouveaux elements sur le mur à piliers phénicien". Transeuphratène 11: 77-94.

Estaca, V. e Yravedra, J. (2013). "Informe Arqueozoológico del Yacimiento de Teatro Cómico", en J.M. Gener, M.A. Navarro y J.M. Pajuelo, Memoria final de la intervención arqueológica puntual en el solar del antiguo Teatro Cómico. Cádiz 2006-2010: 417438. Memoria depositada en la Delegación Territorial de Educación, Cultura y Deporte de la Junta de Andalucía en Cádiz (Reg. exp. A-28/06 (352) y en el Área de Urbanismo del Excmo. Ayuntamiento de Cádiz (Reg., exp. 12-072), Cádiz 2013.

Estévez, J. (1983): "La fauna del Corte 3: aproximación a la fauna del yacimiento de Setefilla", en M. E. Aubet y otros, La Mesa de Setefilla, Lora del Río (Sevilla). Campaña de 1979 (Excavaciones Arqueológicas en España 122): 158-168. Madrid, Ministerio de Cultura.

Estévez, J. y Paz, M.A. (1985): “Análisis faunístico”, en J. L. Escacena y G. de Frutos, "Estratigrafía de la Edad del Bronce en el Monte Berrueco (Medina 
Sidonia, Cádiz)", Noticiario Arqueológico Hispánico 24: 84-85.

Fernandez, H. (2001): Ostéologie comparée des petites ruminants eurasiatiques sauvages et domestiques (genres Rupicapra hircus, Ovis aries, Capra hircus et Capreolus): diagnose differentialle du squelette apendiculaire. Tesis Doctoral, Université de Geneva, Facultat de Ciencies. Pth. Inédita.

Fierro, J.A. (1979): Cádiz la única posibilidad de un Tartessos atlántico. Cádiz, J.A. Fierro.

Fierro, J.A (1983): Opiniones sobre los asentamientos $y$ lugares de culto, Cádiz, J.A. Fierro.

Fierro, J.A (1995): Gadir. La historia de un mito. Cádiz, J.A. Fierro.

Fumadó, I. (2013): “¿Quién parte y reparte? Análisis de la disposición urbana de la Cartago fenicia". Archivo Español de Arqueología 86: 7-21.

Gener, J.Ma; Navarro, M.A.; Pajuelo, J.M.; Torres, M. y Domínguez Bella, S. (2012): "Las crétulas del siglo VIII a.C. de las excavaciones del solar del Teatro Cómico (Cádiz)". Madrider Mitteilungen 53: 134-185.

Gener, J.M ${ }^{\text {a }}$ Navarro, M.A.; Pajuelo, J.M. y Torres, M. (e.p.): "Arquitectura y urbanismo de la Gadir fenicia: el yacimiento del "Teatro Cómico" de Cádiz". Collezione di Studi Fenici. Roma, ISCIMA.

Guadelli, J.L. (1998): "Détermination de l'age des caveaux fossiles et établissement des chasses d'age". Paléo 10: 87-93.

Gutiérrez, J.Ma; Martín, A.; Domínguez-Bella, S. y Moral Cardona, J.P. (1991): Introducción a la geología de la provincia de Cádiz. Cádiz, Universidad de Cádiz.

Helas, S. y Marzoli, D. (eds.) (2009): Phönizisches und punisches Städtwesen. Akten der internationalen Tagung in Rom vom 21.bis 23 Februar 2007, Phönizisches und punisches Städtewesen. Iberia Archaeologica 13: 55-57. Mainz am Rhein, Philipp von Zabern.

Herzog, Z., (2007): Archaeology of the city: Urban planning in Ancient Israel and its social implications. Sidney, Emery and Claire Yass Archaeology Press.

Hilson, S. (1992): Mammal Bones and Teeth: An introductory guide to methods of identification. Londres, London Institute of Archaeology.

Klein, R.G.; Wolf, C.; Freeman, L.G. y Allwayden, K. (1981): "The use of dental crown heights for constructing age profiles of red deer and similar species in archaeological samples". Journal of Archaeological Science 8: 1-31.
Klein, R.G.; Allwayden, K. y Wolf., C. (1983): "The calculation and interpretation of ungulate age profiles from dental crown heights", en G. Bailey (ed.), Hunter gatherer economy in prehistory: a European Perspective. Londres, London University Press.

Lavocat, R. (1966): Faunes et Flores prehistoriques de L'Europe Occidentale. Collection L'homme et ses Origenes. París, IPH.

Levine, M. A. (1982): "The use of crown height measurements and eruption-wear sequence to age horse teeth", en B. Wilson, C. Grigson y S. Payne (eds.), Aging and sexing from archaeological sites. BAR British Series 109: 223-250. Oxford, BAR.

Mariezkurrena, K. (1983): "Contribución al conocimiento del desarrollo de la dentición y el desarrollo del esqueleto postcranial de Cervus elaphus". Munibe 35: 149-202.

Martín Roldán, R. (1959): "Estudio anatómico de los restos óseos procedentes de las excavaciones arqueológicas en el Cerro "El Carambolo" (Sevilla)". Anales de la Universidad Hispalense XIX: 11-47.

Martín Roldán, R. y Blázquez Layunta, M.J. (1983): Apuntes de Osteología Diferencial en Mamíferos. Cátedra de Anatomía y Embriología, Facultad de Veterinaria, Universidad Complutense, Madrid. Madrid, Universidad Complutense Edit.

Mirabent, J. (1850): Memoria sobre las pescas que se cultivan en las costas meridionales de España, desde el cabo de San Vicente hasta el Estrecho de Gibraltar. Huelva, Imprenta de Don José Reyes.

Montero, M. (1999): "Explotación y consumo de animales domésticos y salvajes. Informe de Arqueofauna", en $\mathrm{M}^{\mathrm{a}}$ E. Aubet, P. Carmona, E. Curia, A. Delgado y A. Fernández, Cerro del Villar. I. El asentamiento fenicio en la desembocadura del río Guadalhorce y su interacción con el Hinterland. Arqueología: 313-319. Monografías Junta de Andalucía. Sevilla, Junta de Andalucía.

Morales, A.; Cereijo, M.A.; Brännstom, P. y Liesau, C. (1994): "The mammals", en E. Roselló y A. Morales, (eds.): Castillo de Doña Blanca. Archaeo-environmental inverigations in the Bay of Cádiz, Spain (750-500 B. C.). BAR International Series 593-594: 37-69. Oxford.

Morales, A.; Roselló, E.; Moreno Nuño, R.; Cereijo Pecharromán, M.A. y Hernández Carrasquilla, F. (1995): "Bases de subsistencia de origen animal en el sudoeste peninsular durante el primer milenio", en Tartesos 25 años después 1968-93. Jerez de la Frontera. Publicación Conmemorativa del V Symposium Internacional de Prehistoria 
Peninsular: 523-548. Jerez de la Frontera, Ayuntamiento de Jerez de la Frontera.

Niveau de Villedary y Mariñas, A.M. y Castro Páez, E. (2008): "Banquets rituels dans la necropole punique de Gadir" Food \& History 6(2): 7-46.

Palomo, S. y Arroyo, E. (2011): Estudio del origen étnico a partir del ADN mitocondrial en dos individuos procedentes del solar del antiguo Teatro Cómico, Informe realizado por el Laboratorio de Genética Forense y Genética de Poblaciones. Departamento de Toxicología y Legislación Sanitaria. Facultad de Medicina de la Universidad Complutense de Madrid. Madrid. Memoria depositada en el Área de Urbanismo del Excmo. Ayuntamiento de Cádiz (Reg., exp. 12-072).

Pales, L. y Lambert, C. (1971): Atlas ostéologique pour servir à la identification des mamiferes du quaternaire. París, IPH.

Payne, S. (1985): "Morphological distinction between the Mandibular teeth of young sheep, Ovis aries and goats, Capra hircus". Journal of Archaeological Science 12: 139-147.

Pérez de Ayala, A. (2011a): Estudio de la malacofauna procedente de la excavación del solar del antiguo Teatro Cómico. Cádiz. Memoria depositada en el Área de Urbanismo del Excmo. Ayuntamiento de Cádiz, (Reg., exp. 12-072).

Pérez de Ayala, A. (2011b): Estudio de la ictiofauna procedente de la excavación del solar del antiguo Teatro Cómico de Cádiz. Memoria depositada en el Área de Urbanismo del Excmo. Ayuntamiento de Cádiz, (Reg., exp. 12-072).

Pérez Ripoll, M. (1988): "Estudio de la secuencia del desgaste de los molares de Capra hircus pyrenaica de los yacimientos prehistóricos". Archivo de Prehistoria Levantina 18: 83-128.

Prummel, W. (1988): "Distinguishing features en postcranial skeletal elements of cattle, Bos primigenius f, taurus and red deer, Cervus elaphus en Schiften aus der Archaeologish-Zoologischen Arbeistgroupe". Schleswig-Kiel. Heft 12: 5-52.

Prummel, W. y Frisch, H.J. (1986): “A guide for the distinction of species, sex and body size in bones of sheep and goat". Journal Archaeological Sciencie 13: 567-577.

Ramírez, J.R. (1982): Los primitivos núcleos de asentamiento en la ciudad de Cádiz. Cádiz, Excelentísimo Ayuntamiento de Cádiz.

Riquelme Cantal, J.A. (2001): “Ganadería fenicio-púnica: ensayo crítico de síntesis", en De la mar y de la tierra, producciones y productos fenicio-púnicos. XV Jornadas de Arqueología Fenicio-Púnica: 111119. Ibiza (2000). Ibiza.

Riquelme Cantal, J.A. (2001b): "Estudio de los restos óseos de mamíferos recuperados en la campaña de 1997 en Pocito Chico", en J.A. Ruiz Gil y J.J. López Amador (coords.), Formaciones sociales agropecuarias en la bahía de Cádiz. 5000 años de adaptación ecológica en la Laguna del Gallo, El Puerto de Santa María. Memoria Arqueológica de Pocito Chico I 1997-2001: 177-191. Sanlúcar de Barrameda, Arqueodesarrollo Gaditano S.L.

Sharon, I. (1987): "Phoenician and Greek Ashlar construction techniques at Tel Dor". Bulletin of the American Schools of Oriental Research 267: 21-42.

Schmid, E. (1972): Atlas of Animal Bones for Prehistorians, Archaeologist and Quaternary Geologis". Nueva York, Elsevier Publishing Company.

Uerpmann, H.P. y Uerpmann, M (1973): “Die Tierknochenfunde aus der phönizischen Faktorei von Toscanos und anderen phönizish beeinfluBten Fundorten der provinz Málaga in Südspanien". Studien über frühe Tierknochenfunde von der Iberischen Halbinsel 4: 35-100.

Yasur-Landau, A.; Ebeling, J.R. y Mazow, L.B. (eds.) (2011): Household Archaeology in Ancient Israel and Beyond. Leiden, Brill.

Yravedra, J. (2006): Tafonomía aplicada a la Zooarqueología. Serie Aula Abierta. Madrid, UNED.

Yravedra, J. y Estaca, V. (2013): "Implicaciones de la zooarqueología sobre la economía en la Edad del Hierro". Zona Arqueológica, (en prensa).

Zamora, J.A.; Gener, J.M.; Navarro, M.A.; Pajuelo, J.M. y Torres, M. (2010): "Epígrafes fenicios arcaicos en la excavación del Teatro Cómico de Cádiz (2006-2010)". Rivista di Studi Fenici 38/2: 35-68. 